\title{
Diagrammatic perturbation theory and the pseudogap
}

\author{
P. Monthoux ${ }^{\dagger}$ \\ Cavendish Laboratory, University of Cambridge \\ Madingley Road, Cambridge CB3 OHE, United Kingdom
}

(Dated: October 29, 2018)

\begin{abstract}
We study a model of quasiparticles on a two-dimensional square lattice coupled to Gaussian distributed dynamical molecular fields. We consider two types of such fields, a vector molecular field that couples to the quasiparticle spin-density and a scalar field coupled to the quasiparticle number density. The model describes quasiparticles coupled to spin or charge fluctuations, and is solved by a Monte Carlo sampling of the molecular field distributions. The nonperturbative solution is compared to various approximations based on diagrammatic perturbation theory. When the molecular field correlations are sufficiently weak, the diagrammatic calculations capture the qualitative aspects of the quasiparticle spectrum. For a range of model parameters near the magnetic boundary, we find that the quasiparticle spectrum is qualitatively different from that of a Fermi liquid, in that it shows a double peak structure, and that the diagrammatic approximations we consider fail to reproduce, even qualitatively, the nonperturbative results of the Monte Carlo calculations. This suggests that the magnetic pseudogap induced by a coupling to antiferromagnetic spin-fluctuations and the spin-splitting of the quasiparticle peak induced by a coupling to ferromagnetic spin-fluctuations lie beyond diagrammatic perturbation theory. While a pseudogap opens when quasiparticles are coupled to antiferromagnetic fluctuations, such a pseudogap is not observed in the corresponding charge-fluctuation case for the range of parameters studied, where vertex corrections are found to effectively reduce the strength of the interaction. This suggests that one has to be closer to the border of long-range order to observe pseudogap effects in the charge-fluctuation case than for a spin-fluctuation induced interaction under otherwise similar conditions. The diagrammatic approximations that contain first order vertex corrections show the enhancement of the spin-fluctuation induced interaction and the suppression of the effective interaction in the charge-fluctuation case. However, for the range of model parameters considered here, the multiple spin or charge-fluctuation exchange processes not included in the diagrammatic approximations considered are found to be important, especially for quasiparticles coupled to charge fluctuations.
\end{abstract}

PACS numbers: PACS Nos. 71.27.+a

\section{INTRODUCTION}

The concept of elementary excitations and the diagrammatic perturbation-theoretic methods borrowed from quantum field theory have given us, over the past decades, many powerful insights into the behavior of materials. In a number of cases, however, these concepts and methods don't seem to work. In previous papers ${ }^{1.2}$, we presented results on a nonperturbative extension of the magnetic interaction model, which had until then been extensively used in the context of diagrammatic approaches. These latter applications were successful in many respects: in the Eliashberg approximation, the magnetic interaction model correctly anticipated the pairing symmetry of the Cooper state in the copper oxide superconductors ${ }^{3}$ and is consistent with spin-triplet p-wave pairing in superfluid ${ }^{3} \mathrm{He}$ [for a recent review see, e.g., ref $\left.{ }^{4}\right]$. One also gets the correct order of magnitude of the superconducting and superfluid transition temperature $T_{c}$ when the model parameters are inferred from experiments in the normal state of the above systems. However, in Ref 1 it was found that when the model was treated nonperturbatively and one approached the border of magnetic long-range order, the quasiparticle spectrum showed qualitative changes not captured by the Eliashberg approximation. In Ref 1 , we raised the possibility that these qualitative changes, namely the opening of a pseudogap in the quasiparticle spectrum, were intrinsically nonperturbative in nature. In this paper, we examine this possibility by comparing the nonperturbative results to various kinds of perturbation-theoretic approximations.

The paper is organized as follows. In the next section we describe the model as well as the various perturbationtheoretic approximations to be compared to the Monte Carlo calculations. Section III contains the results of the nonperturbative and diagrammatic calculations. Section IV contains a discussion of the results and finally we give a summary and outlook

\section{MODEL}

The model and its motivation have been extensively discussed in Ref 1 . Here we only give the definitions relevant to the present discussion. We consider particles on a two-dimensional square lattice whose Hamiltonian in the absence of interactions is

$$
\hat{h}_{0}(\tau)=-\sum_{i, j, \alpha} t_{i j} \psi_{i \alpha}^{\dagger}(\tau) \psi_{j \alpha}(\tau)-\mu \sum_{i \alpha} \psi_{i \alpha}^{\dagger}(\tau) \psi_{i \alpha}(\tau)
$$


where $t_{i j}$ is the tight-binding hopping matrix, $\mu$ the chemical potential and $\psi_{i \alpha}^{\dagger}, \psi_{i \alpha}$ respectively create and annihilate a fermion of spin orientation $\alpha$ at site $i$. We take $t_{i j}=t$ if sites $i$ and $j$ are nearest neighbors and $t_{i j}=t^{\prime}$ if sites $i$ and $j$ are next-nearest neighbors.

To introduce interactions between the particles, we couple them to a dynamical molecular (or HubbardStratonovich) field. It is instructive to consider two different types of molecular fields. In the first instance, we consider a vector Hubbard-Stratonovich field that couples locally to the fermion spin density. We also consider the case of a scalar field that couples locally to the fermion number density. This case corresponds to a coupling to charge-fluctuations or, within the approximation we are using here, "Ising"-like magnetic fluctuations where only longitudinal modes are present. The Hamiltonians at imaginary time $\tau$ for particles coupled to the fluctuating exchange or scalar dynamical field are then

$$
\begin{aligned}
& \hat{h}(\tau)=\hat{h}_{0}(\tau)-\frac{g}{\sqrt{3}} \sum_{i \alpha \gamma} \mathbf{M}_{i}(\tau) \cdot \psi_{i \alpha}^{\dagger}(\tau) \sigma_{\alpha \gamma} \psi_{i \gamma}(\tau) \\
& \hat{h}(\tau)=\hat{h}_{0}(\tau)-g \sum_{i \alpha} \Phi_{i}(\tau) \psi_{i \alpha}^{\dagger}(\tau) \psi_{i \alpha}(\tau)
\end{aligned}
$$

where $\mathbf{M}_{i}(\tau)=\left(M_{i}^{x}(\tau), M_{i}^{y}(\tau), M_{i}^{z}(\tau)\right)^{T}$ and $\Phi_{i}(\tau)$ are the real vector exchange and scalar HubbardStratonovich fields respectively, and $g$ the coupling constant. The reason for the choice of an extra factor $1 / \sqrt{3}$ in Eq. (2) becomes clear later.

Since we ignore the self-interactions of the molecular fields, their distribution is Gaussian and given by ${ }^{1.2}$

$$
\begin{aligned}
& \mathcal{P}[\mathbf{M}]=\frac{1}{Z} \exp \left(-\sum_{\mathbf{q}, \nu_{n}} \frac{\mathbf{M}\left(\mathbf{q}, i \nu_{n}\right) \cdot \mathbf{M}\left(-\mathbf{q},-i \nu_{n}\right)}{2 \alpha\left(\mathbf{q}, i \nu_{n}\right)}\right) \\
& Z=\int D \mathbf{M} \exp \left(-\sum_{\mathbf{q}, \nu_{n}} \frac{\mathbf{M}\left(\mathbf{q}, i \nu_{n}\right) \cdot \mathbf{M}\left(-\mathbf{q},-i \nu_{n}\right)}{2 \alpha\left(\mathbf{q}, i \nu_{n}\right)}\right)
\end{aligned}
$$

in the case of a vector exchange molecular field and

$$
\begin{aligned}
& \mathcal{P}[\Phi]=\frac{1}{Z} \exp \left(-\sum_{\mathbf{q}, \nu_{n}} \frac{\Phi\left(\mathbf{q}, i \nu_{n}\right) \Phi\left(-\mathbf{q},-i \nu_{n}\right)}{2 \alpha\left(\mathbf{q}, i \nu_{n}\right)}\right) \\
& Z=\int D \Phi \exp \left(-\sum_{\mathbf{q}, \nu_{n}} \frac{\Phi\left(\mathbf{q}, i \nu_{n}\right) \Phi\left(-\mathbf{q},-i \nu_{n}\right)}{2 \alpha\left(\mathbf{q}, i \nu_{n}\right)}\right)
\end{aligned}
$$

in the case of a scalar Hubbard-Stratonovich field. In both cases $\nu_{n}=2 \pi n T$ since the dynamical molecular fields are periodic functions in the interval $[0, \beta=1 / T]$. The Fourier transforms of the molecular fields are defined as

$$
\begin{aligned}
\mathbf{M}_{\mathbf{R}}(\tau) & =\sum_{\mathbf{q}, \nu_{n}} \mathbf{M}\left(\mathbf{q}, i \nu_{n}\right) \exp \left(-i\left[\mathbf{q} \cdot \mathbf{R}-\nu_{n} \tau\right]\right) \\
\Phi_{\mathbf{R}}(\tau) & =\sum_{\mathbf{q}, \nu_{n}} \Phi\left(\mathbf{q}, i \nu_{n}\right) \exp \left(-i\left[\mathbf{q} \cdot \mathbf{R}-\nu_{n} \tau\right]\right)
\end{aligned}
$$

We consider the case where there is no long-range magnetic or charge order. The average of the dynamical molecular fields must then vanish and their Gaussian distributions Eqs. (46) are completely determined by their variance $\alpha\left(\mathbf{q}, i \nu_{n}\right)$, which we take to be

$$
\alpha\left(\mathbf{q}, i \nu_{n}\right)= \begin{cases}\frac{1}{2} \frac{T}{N} \chi\left(\mathbf{q}, i \nu_{n}\right) & \text { if } \mathbf{M}\left(\mathbf{q}, i \nu_{n}\right) \text { or } \Phi\left(\mathbf{q}, i \nu_{n}\right) \\ & \text { is complex } \\ \frac{T}{N} \chi\left(\mathbf{q}, i \nu_{n}\right) & \text { if } \mathbf{M}\left(\mathbf{q}, i \nu_{n}\right) \text { or } \Phi\left(\mathbf{q}, i \nu_{n}\right) \\ & \text { is real }\end{cases}
$$

where $N$ is the number of allowed wavevectors in the Brillouin zone. Then

$$
\begin{aligned}
\left\langle M_{i}\left(\mathbf{q}, i \nu_{n}\right) M_{j}\left(\mathbf{k}, i \Omega_{n}\right)\right\rangle & =\frac{T}{N} \chi\left(\mathbf{q}, i \nu_{n}\right) \\
& \times \delta_{\mathbf{q},-\mathbf{k}} \delta_{\nu_{n},-\Omega_{n}} \delta_{i, j} \\
\left\langle\Phi\left(\mathbf{q}, i \nu_{n}\right) \Phi\left(\mathbf{k}, i \Omega_{n}\right)\right\rangle & =\frac{T}{N} \chi\left(\mathbf{q}, i \nu_{n}\right) \delta_{\mathbf{q},-\mathbf{k}} \\
& \times \delta_{\nu_{n},-\Omega_{n}}
\end{aligned}
$$

where $\langle\ldots\rangle$ denotes an average over the probability distributions Eq. (4) and Eq. (6) for the vector and scalar cases respectively. In order to compare the scalar and vector molecular fields, we take the same form for their correlation function $\chi\left(\mathbf{q}, i \nu_{n}\right)$ and parametrize it as in Refs ${ }^{5.6}$. In what follows, we set the lattice spacing $a$ to unity. For real frequencies, we have

$$
\chi(\mathbf{q}, \omega)=\frac{\chi_{0} \kappa_{0}^{2}}{\kappa^{2}+\widehat{q}^{2}-i \frac{\omega}{\eta(\widehat{q})}}
$$

where $\kappa$ and $\kappa_{0}$ are the correlation wavevectors or inverse correlation lengths in units of the lattice spacing, with and without strong correlations, respectively. Let

$$
\widehat{q}_{ \pm}^{2}=4 \pm 2\left(\cos \left(q_{x}\right)+\cos \left(q_{y}\right)\right)
$$

We consider commensurate charge fluctuations and antiferromagnetic spin fluctuations, in which case the parameters $\widehat{q}^{2}$ and $\eta(\widehat{q})$ in Eq. (13) are defined as

$$
\begin{aligned}
\widehat{q}^{2} & =\widehat{q}_{+}^{2} \\
\eta(\widehat{q}) & =T_{0} \widehat{q}_{-}
\end{aligned}
$$

where $T_{0}$ is a characteristic temperature.

We also consider the case of ferromagnetic spinfluctuations, where the parameters $\widehat{q}^{2}$ and $\eta(\widehat{q})$ in Eq. (13) are given by

$$
\begin{aligned}
\widehat{q}^{2} & =\widehat{q}_{-}^{2} \\
\eta(\widehat{q}) & =T_{0} \widehat{q}_{-}
\end{aligned}
$$


$\chi\left(\mathbf{q}, i \nu_{n}\right)$ is related to the imaginary part of the response function $\operatorname{Im} \chi(\mathbf{q}, \omega), \mathrm{Eq}$. (13), via the spectral representation

$$
\chi\left(\mathbf{q}, i \nu_{n}\right)=-\int_{-\infty}^{+\infty} \frac{d \omega}{\pi} \frac{\operatorname{Im} \chi(\mathbf{q}, \omega)}{i \nu_{n}-\omega}
$$

To get $\chi\left(\mathbf{q}, i \nu_{n}\right)$ to decay as $1 / \nu_{n}^{2}$ as $\nu_{n} \rightarrow \infty$, as it should, we introduce a cutoff $\omega_{0}$ and take $\operatorname{Im} \chi(\mathbf{q}, \omega)=0$ for $\omega \geq \omega_{0}$. A natural choice for the cutoff is $\omega_{0}=\eta(\widehat{q}) \kappa_{0}^{2}$.

In our model, the single particle Green's function is the average over the probability distributions $\mathcal{P}[\mathbf{M}]$ (Eq. (4)) or $\mathcal{P}[\Phi]$ (Eq. (6) ) of the fermion Green's function in a dynamical vector or scalar field.

$$
\begin{aligned}
\mathcal{G}\left(i \sigma \tau ; j \sigma^{\prime} \tau^{\prime}\right) & =\int D \mathbf{M} \mathcal{P}[\mathbf{M}] G\left(i \sigma \tau ; j \sigma^{\prime} \tau^{\prime} \mid[\mathbf{M}]\right)(20) \\
\mathcal{G}\left(i \sigma \tau ; j \sigma^{\prime} \tau^{\prime}\right) & =\int D \Phi \mathcal{P}[\Phi] G\left(i \sigma \tau ; j \sigma^{\prime} \tau^{\prime} \mid[\Phi]\right)
\end{aligned}
$$

where

$$
G\left(i \sigma \tau ; j \sigma^{\prime} \tau^{\prime} \mid[\mathbf{M}] \text { or }[\Phi]\right)=-\left\langle T_{\tau}\left\{\psi_{i \sigma}(\tau) \psi_{j \sigma^{\prime}}^{\dagger}\left(\tau^{\prime}\right)\right\}\right\rangle
$$

is the single particle Green's function in a dynamical molecular field and is discussed at length in Ref 1 . In evaluating expressions Eqs. (2021) one is summing over all Feynman diagrams corresponding to spin or chargefluctuation exchanges 1.2 .7 . The diagrammatic expansion of the Green's function, Eq. (20) is shown pictorially in Fig. 1. Since in our model no virtual fermion loops are present, there is no fermion sign problem ${ }^{2}$.

In this paper we compare the results of the Monte Carlo simulations to various diagrammatic approximations for the same model. We denote by $\mathcal{G}_{0}\left(\mathbf{p}, i \omega_{n}\right)$ and $\mathcal{G}\left(\mathbf{p}, i \omega_{n}\right)$ the bare and dressed quasiparticle propagators respectively. They are given by

$$
\begin{aligned}
\mathcal{G}_{0}\left(\mathbf{p}, i \omega_{n}\right) & =\frac{1}{i \omega_{n}-\left(\epsilon_{\mathbf{p}}-\mu\right)} \\
\mathcal{G}\left(\mathbf{p}, i \omega_{n}\right) & =\frac{1}{i \omega_{n}-\left(\epsilon_{\mathbf{p}}-\mu\right)-\Sigma\left(\mathbf{p}, i \omega_{n}\right)}
\end{aligned}
$$

where $\Sigma\left(\mathbf{p}, i \omega_{n}\right)$ is the quasiparticle self-energy and $\epsilon_{\mathbf{p}}$ the tight-binding dispersion relation obtained from Fourier transforming the hopping matrix $t_{i j}$ in Eq. (1) and $\mu$ the chemical potential. We consider four approximations to the quasiparticle self-energy $\Sigma\left(\mathbf{p}, i \omega_{n}\right)$ whose diagrammatic representations are shown in Fig. 2.

In Fig. 2a, the self-energy is approximated by first order perturbation theory in the exchange of magnetic or charge fluctuations and denoted $\Sigma^{1 p t}\left(\mathbf{p}, i \omega_{n}\right)$. Fig. $2 \mathrm{~b}$ shows the Eliashberg approximation in which the selfenergy denoted $\Sigma^{1 s c}\left(\mathbf{p}, i \omega_{n}\right)$ is given by the first order self-consistent (or Brillouin-Wigner) perturbation theory.
The expressions for $\Sigma^{1 p t}\left(\mathbf{p}, i \omega_{n}\right)$ or $\Sigma^{1 s c}\left(\mathbf{p}, i \omega_{n}\right)$ in the case of quasiparticles coupled to magnetic or charge fluctuations are identical (this is the reason for our choice of the factor $1 / \sqrt{3}$ in Eq. (2) ) and given by

$$
\begin{aligned}
\Sigma^{1 p t}\left(\mathbf{p}, i \omega_{n}\right) & =g^{2} \frac{T}{N} \sum_{\mathbf{k}, \Omega_{n}} \chi\left(\mathbf{p}-\mathbf{k}, i \omega_{n}-i \Omega_{n}\right) \\
& \times \mathcal{G}_{0}\left(\mathbf{k}, i \Omega_{n}\right) \\
\Sigma^{1 s c}\left(\mathbf{p}, i \omega_{n}\right) & =g^{2} \frac{T}{N} \sum_{\mathbf{k}, \Omega_{n}} \chi\left(\mathbf{p}-\mathbf{k}, i \omega_{n}-i \Omega_{n}\right) \\
& \times \mathcal{G}\left(\mathbf{k}, i \Omega_{n}\right)
\end{aligned}
$$

where $\mathcal{G}_{0}\left(\mathbf{k}, i \Omega_{n}\right)$ and $\mathcal{G}\left(\mathbf{k}, i \Omega_{n}\right)$ are the bare and dressed quasiparticle Green's functions defined in Eq. (23) and Eq. (24) respectively. Fig. 2c shows the diagrammatic expansion corresponding to second order perturbation theory and we denote the self-energy corresponding to that approximation $\Sigma^{2 p t}\left(\mathbf{p}, i \omega_{n}\right)$. The second order selfconsistent approximation to the quasiparticle self-energy, denoted $\Sigma^{2 s c}\left(\mathbf{p}, i \omega_{n}\right)$, is shown diagrammatically in Fig. 2d. The expressions for $\Sigma^{2 p t}\left(\mathbf{p}, i \omega_{n}\right)$ and $\Sigma^{2 s c}\left(\mathbf{p}, i \omega_{n}\right)$ now depend on whether the quasiparticles are coupled to the vector Hubbard-Stratonovich field (magnetic fluctuations) or scalar Hubbard-Stratonovich field (charge fluctuations), because vertex corrections in the two cases do not have the same coefficient or even the same sign. The expressions for $\Sigma^{2 p t}\left(\mathbf{p}, i \omega_{n}\right)$ and $\Sigma^{2 s c}\left(\mathbf{p}, i \omega_{n}\right)$ for quasiparticles coupled to magnetic fluctuations are given by

$$
\begin{aligned}
& \Sigma^{2 p t}\left(\mathbf{p}, i \omega_{n}\right)=g^{2} \frac{T}{N} \sum_{\mathbf{k}, \Omega_{n}} \chi\left(\mathbf{p}-\mathbf{k}, i \omega_{n}-i \Omega_{n}\right) \\
& \times \mathcal{G}_{0}\left(\mathbf{k}, i \Omega_{n}\right) \\
& +g^{2} \frac{T}{N} \sum_{\mathbf{k}, \Omega_{n}} \chi\left(\mathbf{p}-\mathbf{k}, i \omega_{n}-i \Omega_{n}\right) \\
& \times \mathcal{G}_{0}\left(\mathbf{k}, i \Omega_{n}\right) \Sigma^{1 p t}\left(\mathbf{p}, i \omega_{n}\right) \mathcal{G}_{0}\left(\mathbf{k}, i \Omega_{n}\right) \\
& -\frac{1}{3}\left(g^{2} \frac{T}{N}\right)^{2} \sum_{\mathbf{k}, \Omega_{n}} \sum_{\mathbf{k}^{\prime}, \Omega_{n}^{\prime}} \chi\left(\mathbf{p}-\mathbf{k}, i \omega_{n}-i \Omega_{n}\right) \\
& \times \mathcal{G}_{0}\left(\mathbf{k}, i \Omega_{n}\right) \mathcal{G}_{0}\left(\mathbf{k}^{\prime}, i \Omega_{n}^{\prime}\right) \chi\left(\mathbf{k}-\mathbf{k}^{\prime}, i \Omega_{n}-i \Omega_{n}^{\prime}\right) \\
& \times \mathcal{G}_{0}\left(\mathbf{p}-\mathbf{k}+\mathbf{k}^{\prime}, i \omega_{n}-i \Omega_{n}+i \Omega_{n}^{\prime}\right) \\
& \Sigma^{2 s c}\left(\mathbf{p}, i \omega_{n}\right)=g^{2} \frac{T}{N} \sum_{\mathbf{k}, \Omega_{n}} \chi\left(\mathbf{p}-\mathbf{k}, i \omega_{n}-i \Omega_{n}\right) \\
& \times \mathcal{G}\left(\mathbf{k}, i \Omega_{n}\right) \\
& -\frac{1}{3}\left(g^{2} \frac{T}{N}\right)^{2} \sum_{\mathbf{k}, \Omega_{n}} \sum_{\mathbf{k}^{\prime}, \Omega_{n}^{\prime}} \chi\left(\mathbf{p}-\mathbf{k}, i \omega_{n}-i \Omega_{n}\right) \\
& \times \mathcal{G}\left(\mathbf{k}, i \Omega_{n}\right) \mathcal{G}\left(\mathbf{k}^{\prime}, i \Omega_{n}^{\prime}\right) \chi\left(\mathbf{k}-\mathbf{k}^{\prime}, i \Omega_{n}-i \Omega_{n}^{\prime}\right) \\
& \times \mathcal{G}\left(\mathbf{p}-\mathbf{k}+\mathbf{k}^{\prime}, i \omega_{n}-i \Omega_{n}+i \Omega_{n}^{\prime}\right)
\end{aligned}
$$

In the case of the scalar Hubbard-Stratonovich field, or coupling to charge fluctuations, the corresponding expressions are 


$$
\begin{aligned}
\Sigma^{2 p t}\left(\mathbf{p}, i \omega_{n}\right) & =g^{2} \frac{T}{N} \sum_{\mathbf{k}, \Omega_{n}} \chi\left(\mathbf{p}-\mathbf{k}, i \omega_{n}-i \Omega_{n}\right) \mathcal{G}_{0}\left(\mathbf{k}, i \Omega_{n}\right) \\
& +g^{2} \frac{T}{N} \sum_{\mathbf{k}, \Omega_{n}} \chi\left(\mathbf{p}-\mathbf{k}, i \omega_{n}-i \Omega_{n}\right) \mathcal{G}_{0}\left(\mathbf{k}, i \Omega_{n}\right) \\
& \times \Sigma^{1 p t}\left(\mathbf{p}, i \omega_{n}\right) \mathcal{G}_{0}\left(\mathbf{k}, i \Omega_{n}\right) \\
& +\left(g^{2} \frac{T}{N}\right)^{2} \sum_{\mathbf{k}, \Omega_{n}} \sum_{\mathbf{k}^{\prime}, \Omega_{n}^{\prime}} \chi\left(\mathbf{p}-\mathbf{k}, i \omega_{n}-i \Omega_{n}\right) \\
& \times \mathcal{G}_{0}\left(\mathbf{k}, i \Omega_{n}\right) \mathcal{G}_{0}\left(\mathbf{k}^{\prime}, i \Omega_{n}^{\prime}\right) \chi\left(\mathbf{k}-\mathbf{k}^{\prime}, i \Omega_{n}-i \Omega_{n}^{\prime}\right) \\
& \times \mathcal{G}_{0}\left(\mathbf{p}-\mathbf{k}+\mathbf{k}^{\prime}, i \omega_{n}-i \Omega_{n}+i \Omega_{n}^{\prime}\right) \\
\Sigma^{2 s c}\left(\mathbf{p}, i \omega_{n}\right) & =g^{2} \frac{T}{N} \sum_{\mathbf{k}, \Omega_{n}} \chi\left(\mathbf{p}-\mathbf{k}, i \omega_{n}-i \Omega_{n}\right) \mathcal{G}\left(\mathbf{k}, i \Omega_{n}\right) \\
& +\left(g^{2} \frac{T}{N}\right)^{2} \sum_{\mathbf{k}, \Omega_{n}} \sum_{\mathbf{k}^{\prime}, \Omega_{n}^{\prime}} \chi\left(\mathbf{p}-\mathbf{k}, i \omega_{n}-i \Omega_{n}\right) \\
& \times \mathcal{G}\left(\mathbf{k}, i \Omega_{n}\right) \mathcal{G}\left(\mathbf{k}^{\prime}, i \Omega_{n}^{\prime}\right) \chi\left(\mathbf{k}-\mathbf{k}^{\prime}, i \Omega_{n}-i \Omega_{n}^{\prime}\right) \\
& \times \mathcal{G}\left(\mathbf{p}-\mathbf{k}+\mathbf{k}^{\prime}, i \omega_{n}-i \Omega_{n}+i \Omega_{n}^{\prime}\right)
\end{aligned}
$$

In Eqs. (27/28129130), $\mathcal{G}_{0}\left(\mathbf{k}, i \Omega_{n}\right)$ and $\mathcal{G}\left(\mathbf{k}, i \Omega_{n}\right)$ are the bare and dressed quasiparticle Green's functions defined in Eq. (23) and Eq. (24) respectively.

\section{A. Mass renormalization parameter}

The strength of the coupling to the magnetic or charge flucutations can be parametrized by a dimensionless mass renormalization parameter $\lambda_{Z}$, which is defined as

$$
\lambda_{Z}=\frac{\int_{-\infty}^{+\infty} \frac{d \omega}{\pi}<\frac{1}{\omega} g^{2} \operatorname{Im} \chi\left(\mathbf{p}-\mathbf{p}^{\prime}, \omega\right)>_{F S\left(\mathbf{p}, \mathbf{p}^{\prime}\right)}}{<1>_{F S(\mathbf{p})}}
$$

The Fermi surface averages are given by

$$
\begin{aligned}
<\cdots>_{F S(\mathbf{p})} & =\int \frac{d^{d} p}{(2 \pi)^{d}} \cdots \delta\left(\epsilon_{\mathbf{p}}-\mu\right) \\
<\cdots>_{F S\left(\mathbf{p}, \mathbf{p}^{\prime}\right)} & =\int \frac{d^{d} p}{(2 \pi)^{d}} \frac{d^{d} p^{\prime}}{(2 \pi)^{d}} \cdots \\
& \times \delta\left(\epsilon_{\mathbf{p}}-\mu\right) \delta\left(\epsilon_{\mathbf{p}^{\prime}}-\mu\right)
\end{aligned}
$$

In practice, we compute the Fermi surface average with a discrete set of momenta and we replace the delta function by a finite temperature expression

$$
\begin{aligned}
\int \frac{d^{d} p}{(2 \pi)^{d}} & \longrightarrow \frac{1}{N} \sum_{\mathbf{p}} \\
\delta\left(\epsilon_{\mathbf{p}}-\mu\right) & \longrightarrow \frac{1}{T} f_{\mathbf{p}}\left(1-f_{\mathbf{p}}\right)
\end{aligned}
$$

where $f_{\mathbf{p}}$ is the Fermi function. Note that $\frac{1}{T} f_{\mathbf{p}}\left(1-f_{\mathbf{p}}\right) \rightarrow$ $\delta\left(\epsilon_{\mathbf{p}}-\mu\right)$ as $T \rightarrow 0$. We have used $T=0.1 t$ and $N=128^{2}$ in all of our calculations. The finite temperature effectively means that van Hove singularities will be smeared out.

Note that the Fermi surface average that appears in $\lambda_{Z}$, Eq. (31) plays a role similar to that of $\alpha^{2} F(\omega) / \omega$ in the case of phonon mediated superconductivity. One therefore expects $\lambda_{Z} \sim 1$ to indicate the crossover between weak and strong coupling.

\section{RESULTS}

The quasiparticle dispersion relation for the twodimensional square lattice is obtained from Eq. (11). We measure all energies and temperatures in units of the nearest-neighbor hopping parameter $t$. We set the nextnearest-neighbor hopping parameter $t^{\prime}=-0.45 t$. The chemical potential is adjusted so that the electronic band filling is $n=0.9$. The dimensionless parameters describing the molecular field correlations are $g^{2} \chi_{0} / t, T_{0} / t, \kappa_{0}$ and $\kappa$. We chose a representative value for $\kappa_{0}^{2}=12$, and set $T_{0}=0.67 t$ as in the earlier work $\mathrm{k}^{\underline{1}}$. For an electronic bandwidth of $1 \mathrm{eV}, T_{0} \approx 1000^{\circ} \mathrm{K}$. We only consider one value of the coupling constant $g^{2} \chi_{0} / t=2$. In the random phase approximation, the magnetic instability would be obtained for a value of $g^{2} \chi_{0} / t$ of the order of 10 . We consider what happens to the quasiparticle spectrum at a fixed temperature $T=0.25 t$ as the inverse correlation length $\kappa$ changes, as in Ref 1 .

All the calculations were done on a 8 by 8 spatial lattice. In the Monte Carlo calculations we used 41 imaginary time slices, or equivalently 41 Matsubara frequencies for the molecular fields, $\mathbf{M}\left(\mathbf{q}, i \nu_{n}\right)$ and $\Phi\left(\mathbf{q}, i \nu_{n}\right)$ $\left(\nu_{n}=2 \pi n T\right.$, with $\left.n=0, \pm 1, \ldots, \pm 20\right)$. In the diagrammatic calculations, we used between 40 to 60 fermion Matsubara frequencies.

By analytic continuation of the single particle Green's function $\mathcal{G}(\mathbf{k}, \tau)$ one can obtain the quasiparticle spectral function $A(\mathbf{k}, \omega)=-\frac{1}{\pi} \operatorname{Im} \mathcal{G}_{R}(\mathbf{k}, \omega)$ and the tunneling density of states $N(\omega)=\frac{1}{N} \sum_{\mathbf{k}} A(\mathbf{k}, \omega)$, where $\mathcal{G}_{R}(\mathbf{k}, \omega)$ is the retarded single particle Green's function. The imaginary time Monte Carlo data is analytically continued with the Maximum Entropy method ${ }^{8}$, using the same methodology as in the earlier work ${ }^{1}$. We used 10000 Monte Carlo samples grouped into 100 bins of 100 samples each. We always use a flat default model in the Maximum Entropy calculations. To provide a fair comparison between diagrammatic and nonperturbative calculations, one should use the same analytic continuation method (with the same parameters) in all cases. Therefore, we generated 100 noisy measurements by adding Gaussian random noise to the results of the diagrammatic calculations and analytically continued $\mathcal{G}(\mathbf{k}, \tau)=$ $\mathcal{G}_{0}(\mathbf{k}, \tau)+T \sum_{\omega_{n}} e^{-i \omega_{n} \tau}\left(\mathcal{G}\left(\mathbf{k}, i \omega_{n}\right)-\mathcal{G}_{0}\left(\mathbf{k}, i \omega_{n}\right)\right)$ using the Maximum Entropy method as well, with the same default model as in the corresponding analytic continua- 
tion of the Monte Carlo data. The scheme is not perfect, however. While the variance of the Gaussian noise added to the diagrammatic Green's functions was chosen such that the statistical uncertainty of the average over the 100 noisy samples was identical to that in the corresponding Monte Carlo Green's function, the correlations in the errors for different values of $\tau$ present in the Monte Carlo results cannot be easily modeled. The Gaussian random numbers added to the diagrammatic Green's function were therefore taken to be independent of each other, and thus the noise in the diagrammatic and Monte Carlo Green's functions did not have identical statistical properties. In spite of this, the present scheme is almost certainly better than the alternatives.

\section{A. Antiferromagnetic spin-fluctuations}

Figs. 3,4 and 5 show the comparison, for different values of $\kappa^{2}$, between the nonperturbative calculations of the quasiparticle Green's function $\mathcal{G}(\mathbf{k}, \tau)$, spectral function $A(\mathbf{k}, \omega)$ and tunneling density of states $N(\omega)$ and those obtained from the approximations $\Sigma^{1 p t}\left(\mathbf{p}, i \omega_{n}\right)$, $\Sigma^{2 p t}\left(\mathbf{p}, i \omega_{n}\right), \Sigma^{1 s c}\left(\mathbf{p}, i \omega_{n}\right)$, and $\Sigma^{2 s c}\left(\mathbf{p}, i \omega_{n}\right)$ to the quasiparticle self-energy.

Fig. 3 shows our results for $\kappa^{2}=24$. For this value of the inverse correlation length squared, the mass renormalization parameter $\lambda_{Z} \approx 0.05$. The coupling to the antiferromagnetic spin-fluctuations is therefore weak. Not surprisingly, the quasiparticle Green's function, spectral function and tunneling density of states obtained from the various diagrammatic approximation agree well with the Monte Carlo results. At $\mathbf{k}=(\pi, 0)$, the difference between the nonperturbative Green's function $\mathcal{G}((\mathbf{k}, \tau)$ and its diagrammatic approximations is of the order of $0.001 \mathrm{t}$ for all values of $\tau$. There is virtually no difference between the straightforward perturbation-theoretic calculations of the spectral function and their self-consistent counterparts, in first and second order, which is expected for weak coupling. Thus the small difference in the spectral functions $A(\mathbf{k}, \omega)$ at $\mathbf{k}=(\pi, 0)$, seen in Fig $3 \mathrm{~b}$, to the extent that they are not an artifact of the analytic continuation, must come from the vertex corrections. Since the first order spectral functions are slightly sharper than the second order ones, the first order vertex corrections result in an increased spin-fluctuation interaction, as pointed

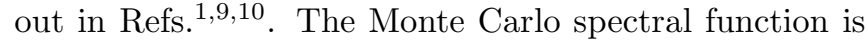
also somewhat broader than the diagrammatic calculations, and provided again that it is not an artifact of the analytic continuation, this suggests that the higher order diagrams lead to a further increase of the spin-fluctuation interaction.

The results for $\kappa^{2}=4$ are shown in Fig. 4. This value of $\kappa^{2}$ gives a mass renormalization parameter $\lambda_{Z} \approx 0.45$. One is now in the intermediate coupling regime. The quasiparticle Green's function $\mathcal{G}(\mathbf{k}, \tau)$ and spectral function $A(\mathbf{k}, \omega)$ at $\mathbf{k}=(\pi, 0)$ as well as the tunneling density of states obtained from the various diagram- matic approximations agree qualitatively with the Monte Carlo results. There are, however, noticeable quantitative differences, not surprisingly much more so than for $\kappa^{2}=24$. The largest difference between the Green's functions obtained from the diagrammatic approximations and the nonperturbative calculations is now bigger than the width of the lines and is roughly an order of magnitude (0.01t) larger than for $\kappa^{2}=24$, which is not unexpected since the mass renormalization parameter is also about an order of magnitude greater for $\kappa^{2}=4$ than for $\kappa^{2}=24$. With the above caveat regarding the analytic continuation, one can make a few additional remarks. First of all, there is now a difference between the straightforward perturbation-theoretic results and the self-consistent calculations of the spectral function $A(\mathbf{k}, \omega)$ at $\mathbf{k}=(\pi, 0)$, both at first and second order. In particular, the second order self-consistent spectral function is slightly broader than the first order selfconsistent one, an indication that the first order vertex correction leads to an enhancement of the effective spinfluctuation interaction, in agreement with Refs $\frac{1.9 .10}{1}$. The nonperturbative $A(\mathbf{k}=(\pi, 0), \omega)$ is broader than the second order self-consistent result, which would imply the higher order vertex corrections are further enhancing the magnetic interaction. Note, that the second order perturbation-theoretic $A(\mathbf{k}=(\pi, 0), \omega)$ is slightly broader than its self-consistent counterpart (the dressing of Green's functions tends to reduce the effect of interactions) and agrees very well with the Monte Carlo result. This may be due to a cancellation of errors (or the analytic continuation procedure) since the agreement between the nonperturbative tunneling density of states $N(\omega)$ and the second order perturbation-theoretic $N(\omega)$ is not as good.

As $\kappa^{2} \approx 1$, the quasiparticle mean free path becomes of the order of the magnetic correlation length for some wavevectors near the Fermi surface, the quasiparticles then can't tell there is no long-range order, and this marks the onset of pseudogap behavior $\stackrel{\underline{1}}{\underline{1}}$. For $\kappa^{2}=1$, the mass renormalization parameter is $\lambda_{Z} \approx 1.1$. One is therefore in the strong coupling regime. The results of our calculations for $\kappa^{2}=1$ are shown in Fig. 5. The developing pseudogap in the spectral function $A(\mathbf{k}, \omega)$ at $\mathbf{k}=(\pi, 0)$ (Fig. 5b) and in the tunneling density of states $N(\omega)$ (Fig. 5e) found in the nonperturbative Monte Carlo calculations is not seen in any of the diagrammatic approximations considered here, which therefore fail qualitatively. Given that one is in the strong coupling regime $\lambda_{Z}>1$, the breakdown of perturbation theory should not come as a surprise. The maximum difference in the quasiparticle Green's function $\mathcal{G}(\mathbf{k}, \tau)$ between the nonperturbative and diagrammatic calculations is now of the order $0.1 \mathrm{t}$, and hence an order of magnitude larger than for $\kappa^{2}=4$ and a couple of orders of magnitude larger than in the weak coupling regime with $\kappa^{2}=24$. It is therefore not suprprising that the quasiparticle spectra that give rise to these rather different imaginary time Green's functions turn out to show qual- 
itative differences. Note that for $\kappa^{2}=1$, there is nearly as much difference between the perturbation-theoretic and self-consistent approximations of the same order as there are between calculations of the same type at first and second order.

\section{B. Ferromagnetic spin-fluctuations}

Figs. 6-9 show our results for the quasiparticle Green's function $\mathcal{G}(\mathbf{k}, \tau)$, spectral function $A(\mathbf{k}, \omega)$ and tunneling density of states $N(\omega)$ for several values of $\kappa^{2}$.

We start with $\kappa^{2}=24$, for which the mass renormalization parameter $\lambda_{Z} \approx 0.05$ for coupling to ferromagnetic spin-flucutations. In this weak coupling regime, Fig. 6 shows that the results of the various diagrammatic calculations are in good agreement with the Monte Carlo results. As in the corresponding antiferromagnetic case, at $\mathbf{k}=(\pi, 0)$, the difference between the nonperturbative Green's function $\mathcal{G}((\mathbf{k}, \tau)$ and its diagrammatic approximations is of the order of $0.001 \mathrm{t}$ for all values of $\tau$. Moreover, there is virtually no difference between the straightforward perturbation-theoretic calculations of the spectral function and their self-consistent counterparts, in first and second order. Thus the small difference in the spectral functions $A(\mathbf{k}, \omega)$ at $\mathbf{k}=(\pi, 0)$, seen in Fig. $6 \mathrm{~b}$ must come from the vertex corrections. Since the first order spectral functions are slightly sharper than the second order ones, the first order vertex corrections result in an increased spin-fluctuation interaction. Given the smallness of the difference between the first and second order results and the ill-posed nature of the analytic continuation problem, one should take the above remark with some degree of caution. In Ref $\frac{\underline{1}}{\underline{1}}$, however, it was shown that the increase in the effective interaction induced by the first order vertex correction is due to the spin dependence of the interaction, and thus should occur for quasiparticles coupled to either antiferromagnetic or ferromagnetic fluctuations. Our analytically continued results are at least consistent with this. From Fig. $6 \mathrm{~b}$, one also sees that the Monte Carlo spectral function is slightly broader than the first or second order results, as in the corresponding antiferromagnetic case. With the above caveat on the nature of the analytic continuation problem, this would suggest the higher order diagrams not included in our perturbation-theoretic approximations lead to a further enhancement of the magnetic interaction, as in the corresponding antiferromagnetic case.

For $\kappa^{2}=4$, the mass renormalization parameter $\lambda_{Z} \approx$ 0.45 and one is therefore in an intermediate coupling regime. Fig. 7 shows that for this value of $\kappa^{2}$, the diagrammatic approximations all qualitatively agree with the Monte Carlo results. The quantitative agreement is, not surprisingly, not as good as in the weak coupling limit with $\kappa^{2}=24$. One notes a number of similarities between the results of Fig. 6 and the corresponding antiferromagnetic case, shown in Fig. 4: (i) the second order perturbation theory results for $A(\mathbf{k}, \omega)$ give the best agreement with the nonperturbative calculation, (ii) since the spectral function in either second order calculation, which include vertex corrections, is slightly broader in $\omega$ than the corresponding first order result, we conclude that first order vertex corrections lead to an enhancement of the effective quasiparticle interaction, which is what is expected on the basis of the arguments made in Refs 1.9 .10 (iii) the spectral function obtained by Monte Carlo sampling of the Gaussian dynamical molecular fields is slightly broader than the second order results, which to the extent this is not an artifact of the Maximum Entropy analytic continuation is an indication that higher order spin-fluctuation exchanges not included in the diagrammatic approximations considered lead to a further enhancement of the effective quasiparticle interaction.

The dynamical exponent $z$ is larger for ferromagnetic than antiferromagnetic spin fluctuations. Hence the effective dimension $d+z=5$ in the ferromagnetic versus $d+z=4$ in the antiferromagnetic case and the standard theory of quantum critical phenomena ${ }^{11}$ leads one to expect weaker corrections for higher effective dimensions. The perturbative calculations qualitatively fail at $\kappa^{2} \approx 1$ in the antiferromagnetic case and on the basis of the above arguments one would expect that the breakdown of perturbation theory in the case of ferromagnetic fluctuations, if it happens, would occur for a smaller value of $\kappa^{2}$ or larger values of the mass renormalization parameter $\lambda_{Z}$. Indeed, at $\kappa^{2}=1, \lambda_{Z} \approx 1.2$ and therefore one is in the strong coupling regime. Fig. 8 shows that while for this value of $\kappa^{2}$ the diagrammatic calculations still agree qualitatively with the Monte Carlo results, unsurprisingly there are larger quantitative differences than in the case $\kappa^{2}=4$ shown in Fig. 7 .

Our results for $\kappa^{2}=0.25$, for which the mass renormalization parameter $\lambda_{Z} \approx 2.3$ are shown in Fig. 9 . The spectral function $A(\mathbf{k}, \omega)$ obtained from the nonperturbative Monte Carlo calculations shows a double peak structure. This has been interpreted in Ref $\frac{1}{m}$ as an effective spin-splitting of the quasiparticle spectrum induced by the local ferromagnetic order. In looking at the evolution of the spectral function $A(\mathbf{k}, \omega)$ as $\kappa^{2}$ is decreased, one first sees a broadening of $A(\mathbf{k}, \omega)$ and then, the broad quasiparticle peak splits into two. The Monte Carlo calculations show very little suppression of the quasiparticle spectral weight or density of states between the two split peaks. A look at Figs. 7c, 8c, and 9e reveals that for $\kappa^{2} \leq 4, N(\omega=0) t \approx 0.15$ and depends very little on $\kappa^{2}$. This is is sharp contrast to the case of antiferromagnetic fluctuations discussed in the previous section. This difference is to be expected of course, since the antiferromagnetic state is gapped while the ferromagnetic state is not. It is clear that none of the diagrammatic approximations considered here reproduce this spin-splitting of the broad quasiparticle peak in $A(\mathbf{k}, \omega)$ and tunneling density of states $N(\omega)$ well. In fact, the first order perturbation theoretic result shows a strong suppression of the tunelling density of states, which clearly doesn't de- 
scribe the precursor to the ferromagnetic state well, and therefore can be considered to fail qualitatively. We observe that the first order perturbation theoretic calculation failed to show a suppression of the tunneling density of states in the antiferromagnetic case where it is obtained in the nonperturbative calculations as expected (see previous subsection) but does show such a pseudogap in the ferromagnetic case where it isn't expected and doesn't appear in the nonperturbative calculations. It therefore qualitatively fails in both cases. Another clear sign that not all is well with the perturbation expansion is the large quantitative differences between the one-loop and two-loop results in Fig.9, something that could be expected at $\lambda_{Z} \approx 2.3$. In the view of the differences between the imaginary time Green's function $\mathcal{G}(\mathbf{k}, \tau)$ obtained from the Monte Carlo simulations and those of the various perturbation-theoretic approximations shown in Figs. 9a and $9 \mathrm{c}$ which are of the order of $0.1 \mathrm{t}$, one would expect the spectral functions that produce these rather different imaginary time Green's functions to be rather different themselves.

\section{Charge fluctuations}

The results of our calculations of the quasiparticle Green's function $\mathcal{G}(\mathbf{k}, \tau)$, spectral function $A(\mathbf{k}, \omega)$ and tunneling density of states $N(\omega)$ for several values of $\kappa^{2}$ are shown in Figs. 10, 11 and 12.

For the model studied here, the mass renormalization parameter $\lambda_{Z}$ is the same for charge and antiferromagnetic fluctuations. Therefore the results of the calculations for $\kappa^{2}=24$ shown in Fig. 10 correspond to $\lambda_{Z} \approx 0.05$, namely the coupling to the charge fluctuations is weak. The agreement between the Monte Carlo results and those of the various diagrammatic approximations is good. As seen in Fig. 10a, the difference between the nonperturbative imaginary time Green's function and its perturbative approximations at $\mathbf{k}=(\pi, 0)$ is less than the width of the line and of the order of $0.001 \mathrm{t}$ for all imaginary times $\tau$. If one compares the results of the perturbation-theoretic calculations at first and second order, one sees from Fig. 10b that there is virtually no difference between the spectral functions $A(\mathbf{k}, \omega)$ obtained by straightforward perturbation theory or the self-consistent calculation at either first or second order. Hence the slight difference bewteen the first and second order calculations, to the extent they aren't an artifact of the analytic continuation, must come from vertex corrections. In contrast to the case of coupling to antiferromagnetic fluctuations, the spectral functions at second order are slightly narrower than their first order counterpart. This suggests the first order vertex correction acts to reduce the effective charge fluctuation interaction, in agreement with the arguments presented in Ref $\underset{1}{\underline{1}}$. Moreover, the nonperturbative $A(\mathbf{k}, \omega)$ at $\mathbf{k}=(\pi, 0)$ is slightly broader than the second order results, which would indicate that the higher order diagrams lead to an enhance- ment of the effective charge fluctuation interaction, as in the case of a coupling to antiferromagnetic fluctuations. While this observation is made on the basis of analytically continued results, it is consistent with the results for other values of $\kappa^{2}$ presented below, where the enhancement of the effective charge fluctuation mediated interaction by higher than second order diagrams can be shown to occur on general grounds.

Fig. 11 shows the the quasiparticle Green's function $\mathcal{G}(\mathbf{k}, \tau)$, spectral function $A(\mathbf{k}, \omega)$ at $\mathbf{k}=(\pi, 0)$ and tunneling density of states $N(\omega)$ for $\kappa^{2}=4$, for which $\lambda_{Z} \approx 0.45$. For this value of $\kappa^{2}$ corresponding to an intermediate coupling regime, the reader will notice that the results of the second order perturbation theory (self-consistent or not) are not displayed in the figures. The reason is that both second order approximations, $\Sigma^{2 p t}\left(\mathbf{p}, i \omega_{n}\right)$ and $\Sigma^{2 s c}\left(\mathbf{p}, i \omega_{n}\right)$, for the model parameters considered here, effectively violate causality requirements in that the Eliashberg renormalization factor $Z\left(\mathbf{p}, i \omega_{n}\right)$ becomes less than one. In terms of the quasiparticle self-energy $\Sigma\left(\mathbf{p}, i \omega_{n}\right), Z\left(\mathbf{p}, i \omega_{n}\right)=1-\frac{1}{\omega_{n}} \operatorname{Im} \Sigma\left(\mathbf{p}, i \omega_{n}\right)=$ $1-\int_{-\infty}^{+\infty} \frac{d \omega}{\pi} \frac{\operatorname{Im} \Sigma_{R}(\mathbf{p}, \omega)}{\omega^{2}+\omega_{n}^{2}}$ where $\operatorname{Im} \Sigma_{R}(\mathbf{p}, \omega)$ is the imaginary part of the retarded self-energy and we have made use of the spectral representation for the self-energy $\Sigma\left(\mathbf{p}, i \omega_{n}\right)=-\int_{-\infty}^{+\infty} \frac{d \omega}{\pi} \frac{\operatorname{Im} \Sigma_{R}(\mathbf{p}, \omega)}{i \omega_{n}-\omega}$. Causality demands that the retarded Green's function be analytic in the upper-half complex frequency plane and therefore that the imaginary part of the retarded self-energy be always less than or equal to zero $\left(\operatorname{Im} \Sigma_{R}(\mathbf{p}, \omega) \leq 0\right)$ for all values of $\mathbf{p}, \omega$. This in turn means that $Z\left(\mathbf{p}, i \omega_{n}\right) \geq 1$ for all values of $\mathbf{p}, \omega$. One can write the second order Eliashberg renormalization factor $Z^{(2)}\left(\mathbf{p}, i \omega_{n}\right)=1+$ $\Delta Z^{(1)}\left(\mathbf{p}, i \omega_{n}\right)+\Delta Z^{(2)}\left(\mathbf{p}, i \omega_{n}\right)$, where $\Delta Z^{(i)}\left(\mathbf{p}, i \omega_{n}\right)$ is the change in $Z\left(\mathbf{p}, i \omega_{n}\right)$ coming from the $i^{t h}$ order diagrams. $\Delta Z^{(1)}\left(\mathbf{p}, i \omega_{n}\right)$ is always greater than zero and therefore poses no problem as far as the condition $Z\left(\mathbf{p}, i \omega_{n}\right) \geq 1$ is concerned. In the charge-fluctuation case, as was ex-

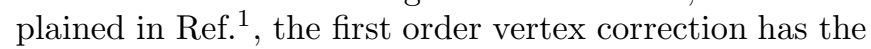
opposite sign compared to the spin-fluctuation case, and leads to a suppression of the effective quasiparticle interaction. The enhancement of the quasiparticle spinfluctuation vertex comes from the transverse magnetic fluctuations that manage to overcome the reduction of the effective coupling due to the longitudinal fluctuations. Because of this cancellation effect, not only is the sign of the first order vertex correction different in the magnetic case, it is also smaller in magnitude than in the charge-fluctuation case, under otherwise similar conditions, as can be seen from the factor $1 / 3$ in Eqs. (2728) not present in the corresponding charge-fluctuation case in Eqs. (2930). The different sign of the vertex corrections in the charge and magnetic cases means that while in the magnetic case $\Delta Z^{(2)}\left(\mathbf{p}, i \omega_{n}\right) \geq 0$ and at second or$\operatorname{der} Z^{(2)}\left(\mathbf{p}, i \omega_{n}\right)$ is always $\geq 1$, in the charge fluctuation case $\Delta Z^{(2)}\left(\mathbf{p}, i \omega_{n}\right) \leq 0$. For $\kappa^{2} \leq 4$, we find that the second order contribution to the Eliashberg renormalization factor is greater in magnitude than the first order con- 
tribution, $\left|\Delta Z^{(2)}\left(\mathbf{p}, i \omega_{n}\right)\right|>\left|\Delta Z^{(1)}\left(\mathbf{p}, i \omega_{n}\right)\right|$ and since it has the opposite sign, $Z^{(2)}\left(\mathbf{p}, i \omega_{n}\right)=1+\Delta Z^{(1)}\left(\mathbf{p}, i \omega_{n}\right)+$ $\Delta Z^{(2)}\left(\mathbf{p}, i \omega_{n}\right) \leq 1$. Note that the nonperturbative calculations always satisfy $Z\left(\mathbf{p}, i \omega_{n}\right) \geq 1$, and the problem only arises in the perturbative approximation and is a sign that, for $\kappa^{2} \leq 4$, the perturbation expansion for the charge-fluctuation case is quite badly behaved, possibly even more so than for magnetic fluctuations. Aso, the fact that the nonperturbative calculations always satisfy $Z\left(\mathbf{p}, i \omega_{n}\right) \geq 1$ is a proof that the higher than second order diagrams contribute to an enhancement of the chargefluctuation interaction for these values of $\kappa^{2}$.

Fig. 11b shows that the spectral function $A(\mathbf{k}, \omega)$ obtained from the nonperturbative calculations is noticeably sharper than those produced by the first order selfconsistent calculations. This means that for $g^{2} \chi_{0} / t=2$ and $T_{0}=0.67 t$ the first and higher order vertex corrections suppress the effective quasiparticle interaction. It is therefore not surprising that there are no qualitative differences between the nonperturbative and diagrammatic calculations. There are quantitative differences, however, and these are more pronounced than in the case of a coupling of quasiparticles to antiferromagnetic spinfluctuations for the same value of $\lambda_{Z}$ shown in Fig. 4.

Finally, Fig. 12 shows our results for $\kappa^{2}=0.25$, for which $\lambda_{Z} \approx 1.8$, hence in the strong coupling regime. The difference between the nonperturbative imaginary time Green's function and its first order diagrammatic approximations seen in Figs. 12b and 12d is a clear indication of the breakdown of perturbation theory. But even in this strong coupling regime, a CDW-precursor pseudogap in the spectral function $A(\mathbf{k}, \omega)$, which can be expected to occur on general grounds 1 is not seen. The pseudogap effects in the charge fluctuation case thus require a stronger coupling still (larger coupling constant $g^{2}$ or smaller value of $\kappa^{2}$ ).

\section{DISCUSSION}

In Ref $\stackrel{1}{\underline{*}}$, we showed that the magnetic pseudogap induced by a coupling to antiferromagnetic spinfluctuations and the spin-splitting of the quasiparticle peak induced by a coupling to ferromagnetic spin fluctuations were not captured by the first order self-consistent, or Eliashberg, approximation. The main result of this paper, is that these phenomena also lie beyond the two magnetic-fluctuation exchange theories (self-consistent or not), which contain first order vertex corrections. While this does obviously not constitute a proof, these results are consistent with the conjecture expressed in Ref. ${ }^{1}$ that the pseudogap effects found in the Monte Carlo calculations are intrinsically nonperturbative in nature. Since the calculations reported here show that the first order vertex corrections alone do not produce a magnetic pseudogap, the physics of that state must then mainly come from the higher order spin-fluctuation exchange processes. The results presented here and in Ref ${ }^{\underline{\underline{\underline{w}}}}$ also indicate that a CDW pseudogap induced by coupling to the scalar dynamical molecular field (Eq. (3)) must also originate from high order charge-fluctuation exchange processes. Close enough to a second order CDW transition, the diverging CDW correlation length is bound to exceed the characteristic length scale for quasiparticles and the calculations of Ref ${ }^{12}$ showed that when this happens a pseudogap opens in the quasiparticle spectrum. The first order vertex correction can't produce the pseudogap state, since as we have seen, in the case of charge fluctuations it leads to a suppression of the interaction. In fact we even found that for the range of model parameters considered here, the second order diagrams more than cancel the contribution from the first order terms leading to a second order Eliashberg renormalization parameter $Z\left(\mathbf{p}, i \omega_{n}\right) \leq 1$, which is inconsistent with causality requirements. Moreover, we expect this "over-cancellation" effect to get worse as $\kappa^{2}$ gets smaller than the lowest value considered here, $\kappa^{2}=0.25$. Since $Z\left(\mathbf{p}, i \omega_{n}\right)$ must be $\geq 1$ when all the diagrams are summed up, as in the Monte Carlo simulations, one can conclude that the higher than second order terms must give a contribution $\Delta Z$ to $Z$ which is positive. Therefore, higher order charge-fluctuation exchange processes produce an enhancement of the effective quasiparticle interaction, as in the magnetic case, and it must be through this enhancement of the effective interaction that a pseudogap can appear in the quasiparticle spectrum on the border of long-range CDW order.

These observations lead one to a unified picture of the pseudogap state found in our model of quasiparticles coupled to spin or charge fluctuations. When the dynamical molecular field correlation length exceeds the characteristic length scale for quasiparticles, either the thermal de Broglie wavelength ${ }^{12,13,14}$ or mean free path ${ }^{1}$, the quasiparticles effectively see long-range order and this marks the onset of the pseudogap state. This state must be produced by high order spin or charge-fluctuation exchanges which contain subtle quantum mechanical coherence effects. In the magnetic fluctuation case, the first order vertex correction favors the pseudogap state, while in the charge fluctuation case it suppresses it. This implies one has to be closer to the boundary of long-range charge order to observe a pseudogap than one has to be to the boundary of magnetic long-range order, under otherwise similar conditions. As the dynamical molecular field correlation length increases, the mass renormalization parameter $\lambda_{Z}$ gets larger, and therefore the many-body effects become stronger. Our results show that the agreement between the results of the Monte Carlo simulations and the perturbation-theoretic results gets worse as $\lambda_{Z}$ increases, and that not surprisingly, the perturbation-theoretic calculations break down when one enters the strong coupling regime $\lambda_{Z}>1$, where the pseudogap is found. A more rigourous analysis of the relevance of the effective quasiparticle interactions as $\kappa^{2}$ increases or as the energy scales are decreased would require a renormalization group $(\mathrm{RG})$ treatment $\mathrm{t}^{15}$. Re- 
cent RG calculations ${ }^{16}$ on the border of the ferromagnetic state indicate that the quasiparticle interactions are indeed relevant in $d \leq 3$, and the RG flows to strong coupling as the energy cutoff is decreased.

One would like to understand what property of the full vertex function $\Gamma_{\alpha, \gamma}^{i}\left(\mathbf{x}, \tau ; \mathbf{x}^{\prime}, \tau^{\prime} \mid \mathbf{x}^{\prime \prime}, \tau^{\prime \prime}\right)=<$ $\left.T_{\tau} \psi_{\alpha}(\mathbf{x}, \tau) \psi_{\gamma}^{\dagger}\left(\mathbf{x}^{\prime}, \tau^{\prime}\right) M^{i}\left(\mathbf{x}^{\prime \prime}, \tau^{\prime \prime}\right)\right\}>$ is responsible for the appearance of the pseudogap and seems to be missing in the first order approximation to $\Gamma_{\alpha, \gamma}^{i}\left(\mathbf{x}, \tau ; \mathbf{x}^{\prime}, \tau^{\prime} \mid \mathbf{x}^{\prime \prime}, \tau^{\prime \prime}\right)$.

Our physical picture of the pseudogap state emerging from quantum mechanical coherence effects contained in high order Feynman diagrams is to be contrasted with the results of Refs $\frac{17.18 .19}{19}$ where a suppression of the quasiparticle tunneling density of states at the Fermi level is obtained in the single spin or charge-fluctuation exchange approximation. This effect is typically obtained with relatively large magnetic or charge correlation lengths. In our model, the calculations reported here and in Ref $\underline{\underline{1}}$ show that as one approaches the border of magnetic longrange order, $\kappa^{2} \rightarrow 0$, the multiple spin-fluctuation exchange processes become important long before a suppression of the quasiparticle tunneling density of states at the Fermi level is seen in the first order perturbationtheoretic and self-consistent calculations. Indeed, pseudogap effects are only obtined in our calculations when the dimensionless mass renormalization parameter $\lambda_{Z}>$ 1 , i.e in the strong coupling regime where one doesn't expect diagrammatic perturbation theory to give reliable approximations. The above finding is likely to be valid more generally, since the intuitive arguments for the physical origin of the pseudogap 1.12.13.14 lead one to expect the breakdown of Migdal's theorem to be a generic occurence near a spin or charge instability. There is also an important difference between a vertex correction induced pseudogap and a single-fluctuation exchange pseudogap. In the latter case, there is no essential distinction bewteen spin and charge fluctuations, in that at the single-fluctuation exchange level, for a given fluctuation spectrum the spin and charge-fluctuation theories of the quasiparticle spectral function can be made identical by an appropriate scaling of the coupling constant to the molecular field. This is no longer the case when vertex corrections are included, since these actually depend on the nature of the Hubbard-Stratonovich field, in our case vector versus scalar. The distinction could turn out to be essential, since we find, for a range of model parameters, that a pseudogap is observed for quasiparticles coupled to spin fluctuations but not in the corresponding chargefluctuation case.

Moukouri et al ${ }^{14}$ have developed a many-body theory of the precursor pseudogap to the Mott transition in the half-filled Hubbard model. Their theory is inspired by the fluctuation exchange approximation (FLEX) ${ }^{20}$ in which bare spin and charge susceptibilities are used to build up the effective quasiparticle interaction, corresponding to $g^{2} \chi(\mathbf{q}, \omega)$ in our model. The key respect in which the theory of Moukouri et al $\stackrel{14}{\underline{14}}$ differs from FLEX is that the coupling to spin and charge fluctuations are not given by the bare on-site Coulomb repulsion, but by renormalized parameters determined self-consistently in such a way that an exact relationship between the single and twoparticle Green's functions is satisfied. This last step goes beyond perturbation theory and it is therefore plausible that the precursor pseudogap to the Mott transition seen in the Monte Carlo simulations of the half-filled Hubbard mode ${ }^{14}$ is also nonperturbative in origin. The analog of their scheme for the present model would be the use of the first order perturbation theory approximation for the quasiparticle self-energy $\Sigma^{(1 p t)}(\mathbf{p}, \omega)$, Eq. (25) and a simultaneous renormalization of the coupling constant $g$ and the correlation wavevector $\kappa^{2}$. A renormalization of the coupling constant $g$ could account for all vertex corrections provided they are local in space and time.

One can indeed get a pseudogap in the tunneling density of states with the first order perturbation theory approximation to $\Sigma(\mathbf{p}, \omega)$ (Eq. (25)), as in Refs ${ }^{17.18}$, provided $\kappa^{2}$ is renormalized to lower values and $g$ renormalized to higher ones. One would thus have to renormalize the model to stronger coupling, roughly to values of $\lambda_{Z} \sim 5$. It should be clear that in this regime, first order perturbation theory is not controlled. One would also naively expect a sensible renormalization scheme that goes beyond the one-loop level to lead to renormalized values of $\kappa^{2}$ larger than the bare value. The renormalized theory should be further away from the magnetic instability than the one-loop approximation rather than closer to it, since ideally one would like the improved theory to satisfy the Mermin-Wagner theorem in two dimensions. If $\kappa^{2}$ were to be increased by the renormalization scheme, in order to obtain a pseudogap in $N(\omega)$ one would likely need a large renormalization of the coupling $g$ and such a scheme for the present model does not look promising to the author. However, it is important to note that the model studied here, although similar in some respects, is actually different than the one considered in Ref 14 . The renormalization scheme proposed by Moukouri et al. which works well for the Hubbard model need not necessarily apply to other theories.

In Ref $\frac{1}{1}$, we pointed out that in the case of quasiparticles coupled to ferromagnetic spin fluctuations, our results are at variance with expectations based on the standard theory of quantum critical phenomena11. Since the dynamical exponent $z=3$, in $d=2$ spatial dimensions, the effective dimension is $d+z=5$ and is greater than the upper critical dimension $d_{c}=4$ above which one would expect the first order theory to be at least qualitatively correct. But our nonperturbative results show that at least for small enough $\kappa^{2}$, the first order theory qualitatively breaks down. Pepin et. $\mathrm{a}^{16}$ have shown that the quasiparticle interactions are indeed relevant in the RG sense for ferromagnetic fluctuations in $d \leq 3$, a result consistent with our findings. For antiferromagnetic and charge fluctuations, $d+z=4$, the marginal case, and hence the qualitative breakdown of the first order approximation isn't necessarily inconsistent with the standard theory. However, the scaling relations derived in Ref ${ }^{11}$ 
rely on the applicability of perturbation theory. If the pseudogap effects are indeed intrinsically nonperturbative in nature, a conjecture that is consistent with the present work, it opens the possibility that the physics in the proximity of a quantum critical point is dominated by nonperturbative quantum mechanical effects and therefore even richer than anticipated in the earlier work ${ }^{11}$. A number of new ideas in this field have recently been proposed ${ }^{21.22}$ and a discussion of some fundamental problems associated with quantum critical points can be found in $\operatorname{Ref}^{23}$.

\section{OUTLOOK}

We studied a nonperturbative formulation of the magnetic interaction model, in which quasiparticles are coupled to a Gaussian distributed dynamical molecular exchange field. Far from the magnetic boundary, the multiple magnetic fluctuation exchange processes do not bring about qualitative changes to the quasiparticle spectrum. But as one gets closer to the border of long-range magnetic order, we find, for a range of model parameters, that Migdal's theorem doesn't apply and the quasiparticle spectrum is qualitatively different from its Eliashberg approximation. Moreover, we find that going one step beyond the single spin-fluctuation exchange approximation and including first order vertex corrections, selfconsistently or not, doesn't help to reproduce the qualitative changes seen in the nonperturbative calculations. Near the magnetic boundary, the simple perturbation expansion shows signs it is not well behaved, since the second order results differ greatly from their first order counterparts. The self-consistent, or renormalized perturbation expansion, which effectively consists in a reordering of the diagrammatic perturbation theory, is better behaved in that the differences between first and second order are much less pronounced. However, even if the renormalized perturbation expansion converges, our re- sults show that it is quite likely to converge to the wrong answer, which could be explained if the original perturbation expansion is divergent.

The intuitive argument for the onset of pseudogap behavior ${ }^{1.12 .13 .14}$, namely that if the distance quasiparticles can travel during their lifetime becomes shorter than the molecular field correlation length, these quasiparticles effectively see long-range order, does not explain the failure of the single spin-fluctuation exchange approximation. As we pointed out in Ref $\frac{1}{1}$, one can get in the regime where the mean-free path gets shorter than $1 / \kappa$ in the Eliashberg approximation, but fail to observe a pseudogap in this regime. Since we have not been able to produce a good fit to the Monte Carlo simulations by including either first order or vertex corrections that are local in space and time, i.e by a renormalization of the coupling constant $g$ to the molecular field, we conjecture that the physical origin of the pseudogap state found in the present calculations lies in non-local vertex corrections produced by high order spin-fluctuation exchanges. These vertex corrections effectively induce a quasiparticle coupling to the dynamical molecular field that is non-local in both space and time. The above conjecture raise the question of what essential property of the vertex function is not captured by its first order approximation. A study of the vertex function along the same lines as the work reported here for the single particle Green's function should provide further insights into this problem.

\section{ACKNOWLEDGMENTS}

I would like to thank P. Coleman, J.R. Cooper, P.B. Littlewood, G.G. Lonzarich, J. Loram, and D. Pines for discussions on this and related topics. We acknowledge the support of the EPSRC, the Newton Trust and the Royal Society.
$0 \dagger$ Present address: School of Physics and Astronomy and Centre for Science at Extreme Conditions, University of Edinburgh, Edinburgh, EH9 3JZ.

1 P. Monthoux, Phys. Rev. B 68, 064408 (2003).

2 P. Monthoux, Phil. Mag. B 79, 15 (1999).

3 N.E. Bickers, D.J. Scalapino, and S.R. White, Phys. Rev. Lett. 62, 961 (1989); T. Moriya, Y. Takahashi and K. Ueda, J. Phys. Soc. Jpn. 52, 2905 (1990); P. Monthoux, A.V. Balatsky and D. Pines, Phys. Rev. Lett. 67, 3448 (1991).

4 E.R. Dobbs, "Helium Three", Oxford University Press (2001).

5 P. Monthoux and G.G. Lonzarich, Phys. Rev. B 59, 14598 (1999).

6 P. Monthoux and G.G. Lonzarich, Phys. Rev. B 63, 054529 (2001).

7 A. Posazhennikova and P. Coleman, Phys. Rev. B 67,
165109 (2003).

8 M. Jarrell and J.E. Gubernatis, Phys. Rep. 269, 134 (1996).

9 P. Monthoux, Phys. Rev. B 55, 15261 (1997).

10 A.V. Chubukov, P. Monthoux and D.R. Morr, Phys. Rev. B 56, 7789 (1997).

11 J.A. Hertz, Phys. Rev. B 14, 1165 (1976); A.J. Millis, Phys. Rev. B 48, 7183 (1993).

12 P. Monthoux and D.J. Scalapino, Phys. Rev. B 65, 235104 (2002).

13 Y.M. Vilk and A.-M. S. Tremblay, J. Phys. I, France 7, 1309 (1997).

14 S. Moukouri, S. Allen, F. Lemay, B. Kyung, D. Poulin. Y. M. Vilk and A.-M. S. Tremblay, Phys. Rev. B 61, 7887 (2000).

15 R. Shankar, Rev. Mod. Phys. 66, 129, (1994); M. Salmhofer and C. Honerkamp, Prog. Theor. Phys. 105, 
$1(2001)$

16 C. Pépin, J. Rech and R. Ramazashvili, cond-mat/0210208

17 A.P. Kampf and J.R. Schrieffer, Phys. Rev. B 41, 6399 (1990).

18 P. Prelovsek and R. Ramsak, Phys. Rev. B 65, 174529 (2002).

19 P.A. Lee, T.M. Rice and P.W. Anderson, Phys. Rev. Lett. 31, 462 (1973).

20 N.E. Bickers and D.J. Scalapino, Ann. Phys. (N.Y.) 193, 206 (1989).

21 P. Coleman and C. Pépin, Physica B 312, 383 (2002).

22 Q.M. Si, S. Rabello, K. Ingersent, J.L. Smith, Nature 413, 804 (2001).

${ }^{23}$ R.B. Laughlin, G.G. Lonzarich, P. Monthoux and D. Pines, Adv. Phys. 50, 361 (2001).

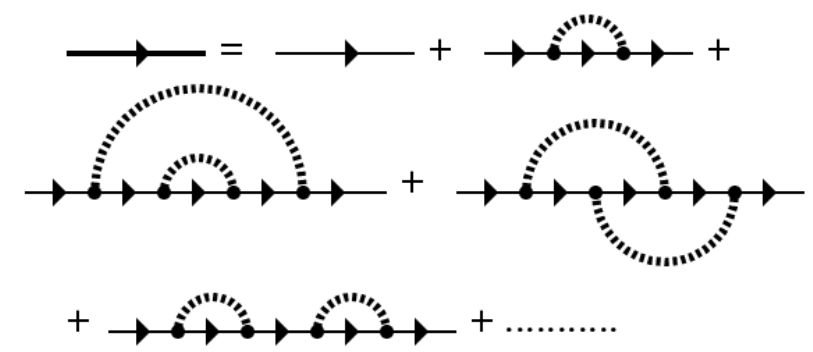

FIG. 1: Diagrammatic expansion for the single particle Green's function, Eq. 201 up to two spin or charge fluctuation exchanges. The dashed line represents the dynamical susceptibility $\chi\left(\mathbf{q}, i \nu_{n}\right)$. (a)

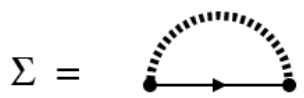

(b)

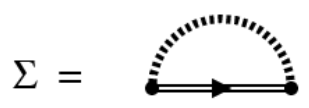

(c)

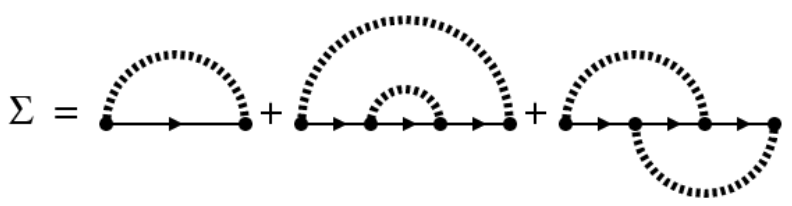

(d)

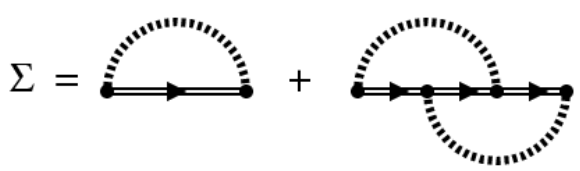

FIG. 2: The various perturbation-theoretic approximations to the quasiparticle self-energy considered in this paper. (a) First order perturbation theory. (b) First order self-consistent (Eliashberg). (c) Second order perturbation theory (d) Second order self-consistent. In this figure a single line denotes the bare quasiparticle propagator $G_{0}$ while a double line denotes the dressed propagator $G$ to be determined selfconsistently. 

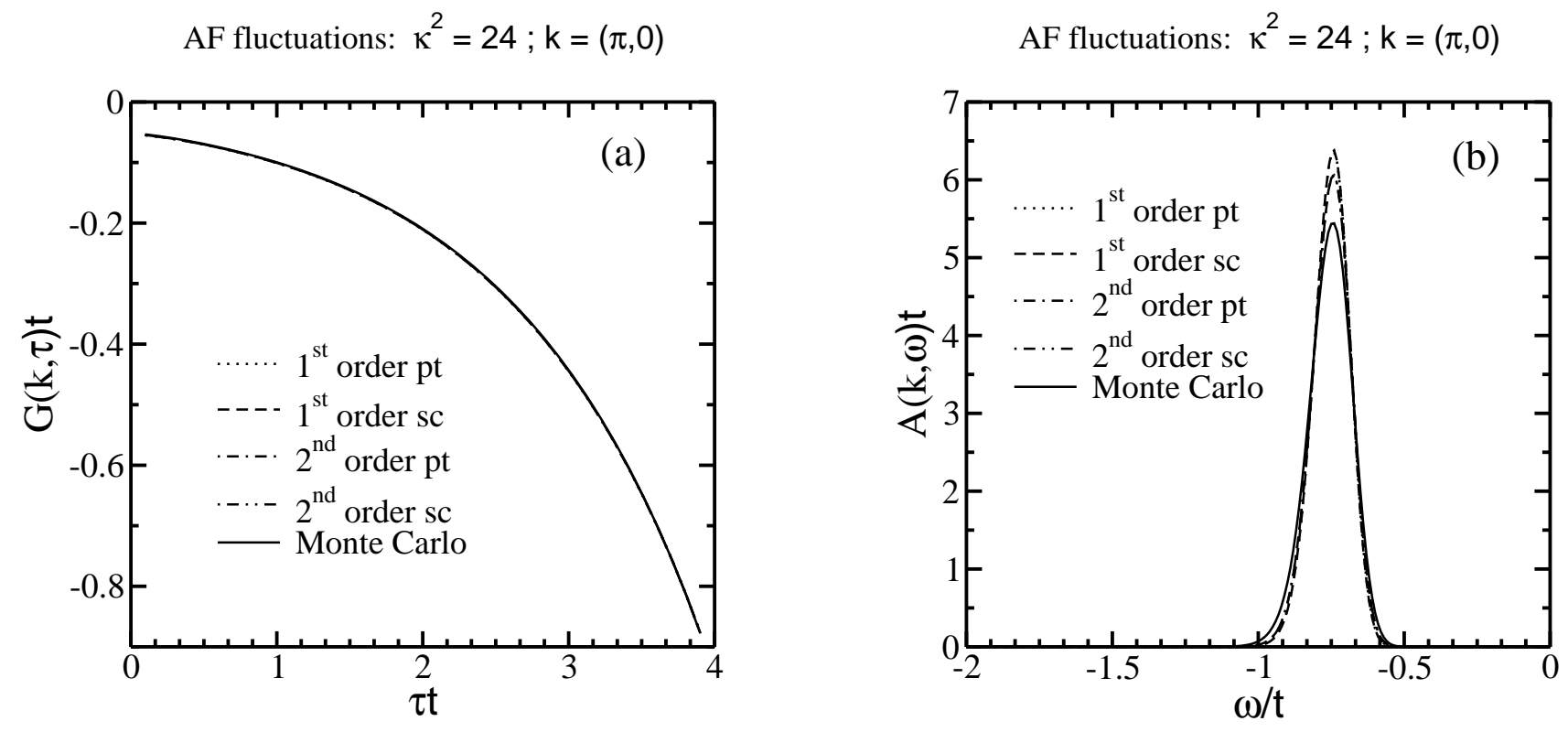


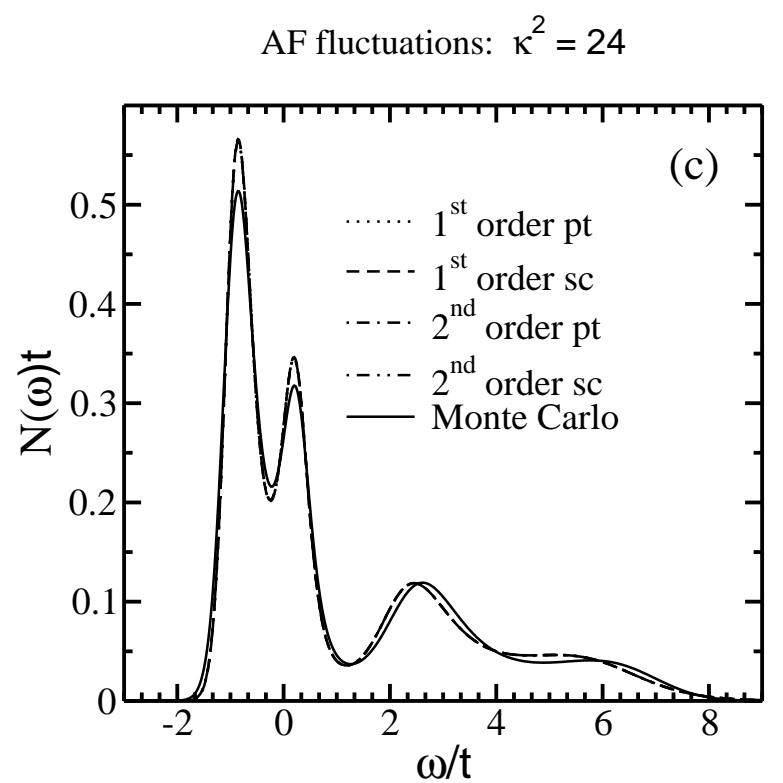

FIG. 3: The various perturbation-theoretic approximations to the quasiparticle imaginary time Green's function $\mathcal{G}(\mathbf{k}, \tau)$, spectral function $A(\mathbf{k}, \omega)$ and tunneling density of states $N(\omega)$ are compared to the results of the Monte Carlo simulations for $\kappa^{2}=24$ in the case of quasiparticles coupled to antiferromagnetic spin-fluctuations. $1^{\text {st }}$ order pt corresponds to the approximation to the self-energy shown in Fig. 2a and given by Eq. 25). $2^{\text {nd }}$ order pt corresponds to the approximation to the self-energy shown in Fig. 2c and given by Eq. (27). $1^{\text {st }}$ order sc corresponds to the approximation to the self-energy shown in Fig. 2b and given by Eq. (26). $2^{\text {nd }}$ order sc corresponds to the approximation to the self-energy shown in Fig. $2 \mathrm{~d}$ and given by Eq. (28). The error bars on the Monte Carlo imaginary time Green's function are not shown for clarity. They are of the order of 0.00002 t. (a) $\mathcal{G}(\mathbf{k}, \omega)$ at $\mathbf{k}=(\pi, 0)$. (b) $A(\mathbf{k}, \omega)$ at $\mathbf{k}=(\pi, 0)$. (c) $N(\omega)$. 

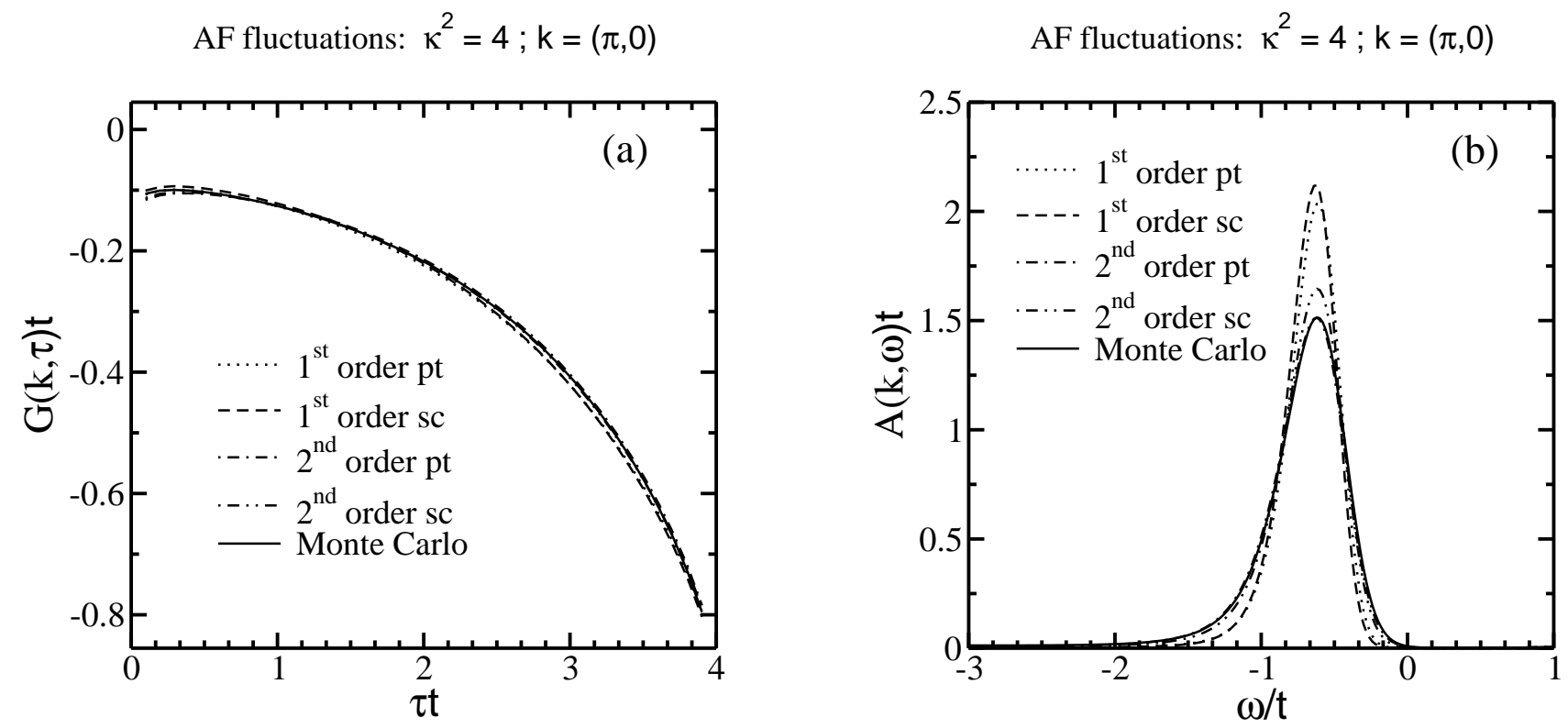


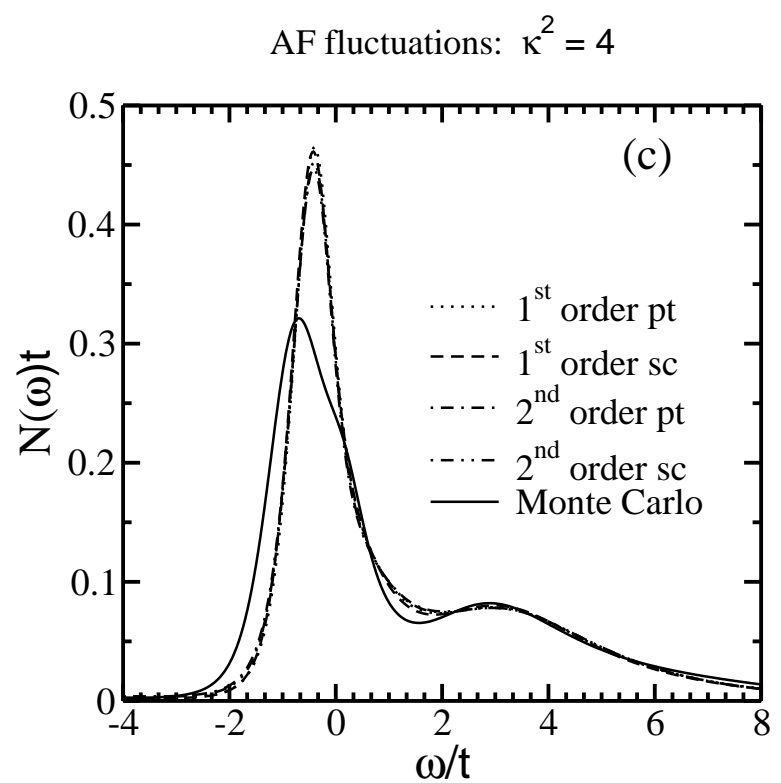

FIG. 4: The various perturbation-theoretic approximations to the quasiparticle imaginary time Green's function $\mathcal{G}(\mathbf{k}, \tau)$, spectral function $A(\mathbf{k}, \omega)$ and tunneling density of states $N(\omega)$ are compared to the results of the Monte Carlo simulations for $\kappa^{2}=4$ in the case of quasiparticles coupled to antiferromagnetic spin-fluctuations. $1^{\text {st }}$ order pt corresponds to the approximation to the self-energy shown in Fig. 2a and given by Eq. (25). $2^{\text {nd }}$ order pt corresponds to the approximation to the self-energy shown in Fig. 2c and given by Eq. (27). $1^{\text {st }}$ order sc corresponds to the approximation to the self-energy shown in Fig. 2b and given by Eq. (26). $2^{\text {nd }}$ order sc corresponds to the approximation to the self-energy shown in Fig. $2 \mathrm{~d}$ and given by Eq. (28). The error bars on the Monte Carlo imaginary time Green's function are not shown for clarity. They are of the order of 0.00015 t. (a) $\mathcal{G}(\mathbf{k}, \omega)$ at $\mathbf{k}=(\pi, 0)$. (b) $A(\mathbf{k}, \omega)$ at $\mathbf{k}=(\pi, 0)$. (c) $N(\omega)$. 

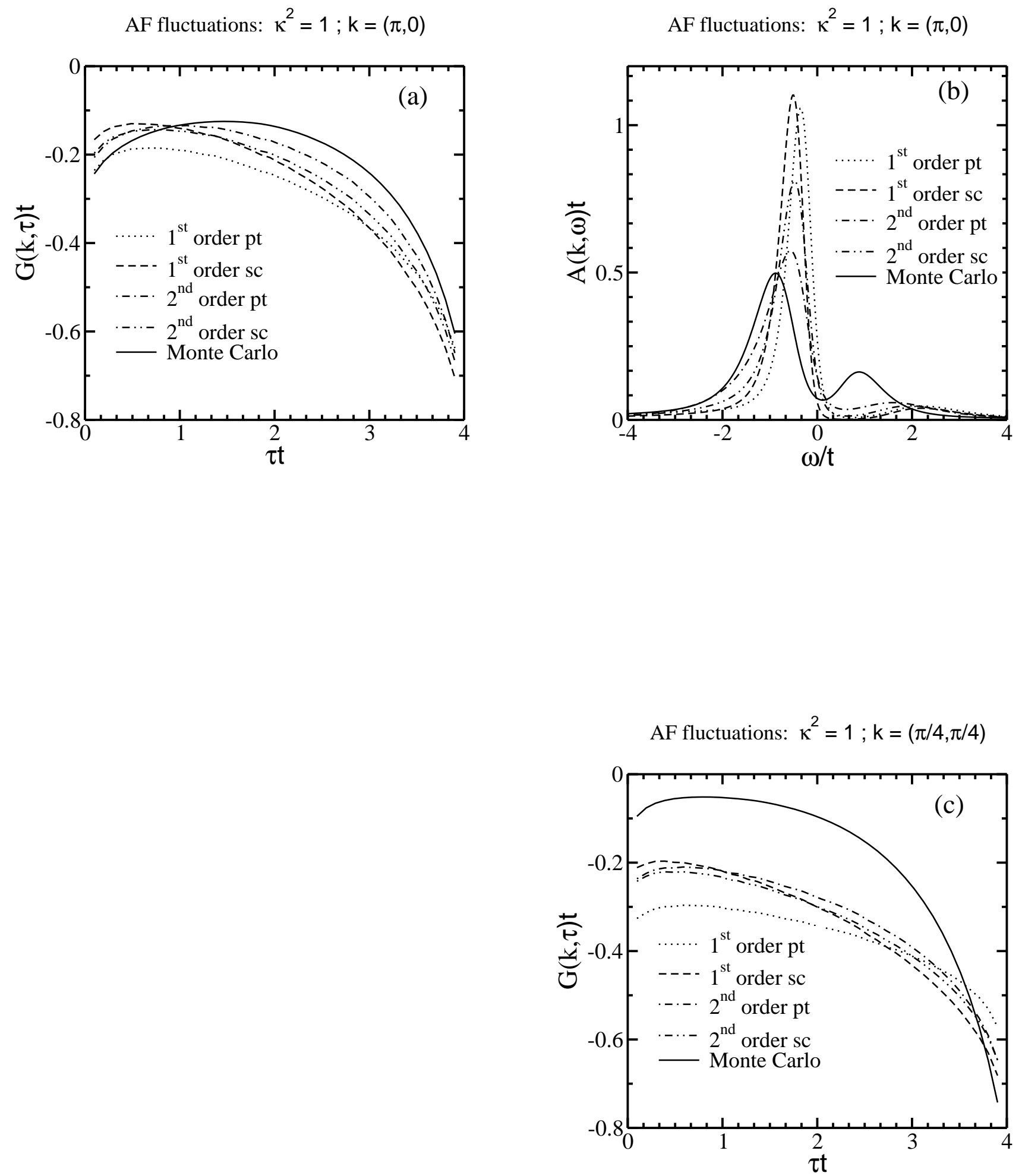

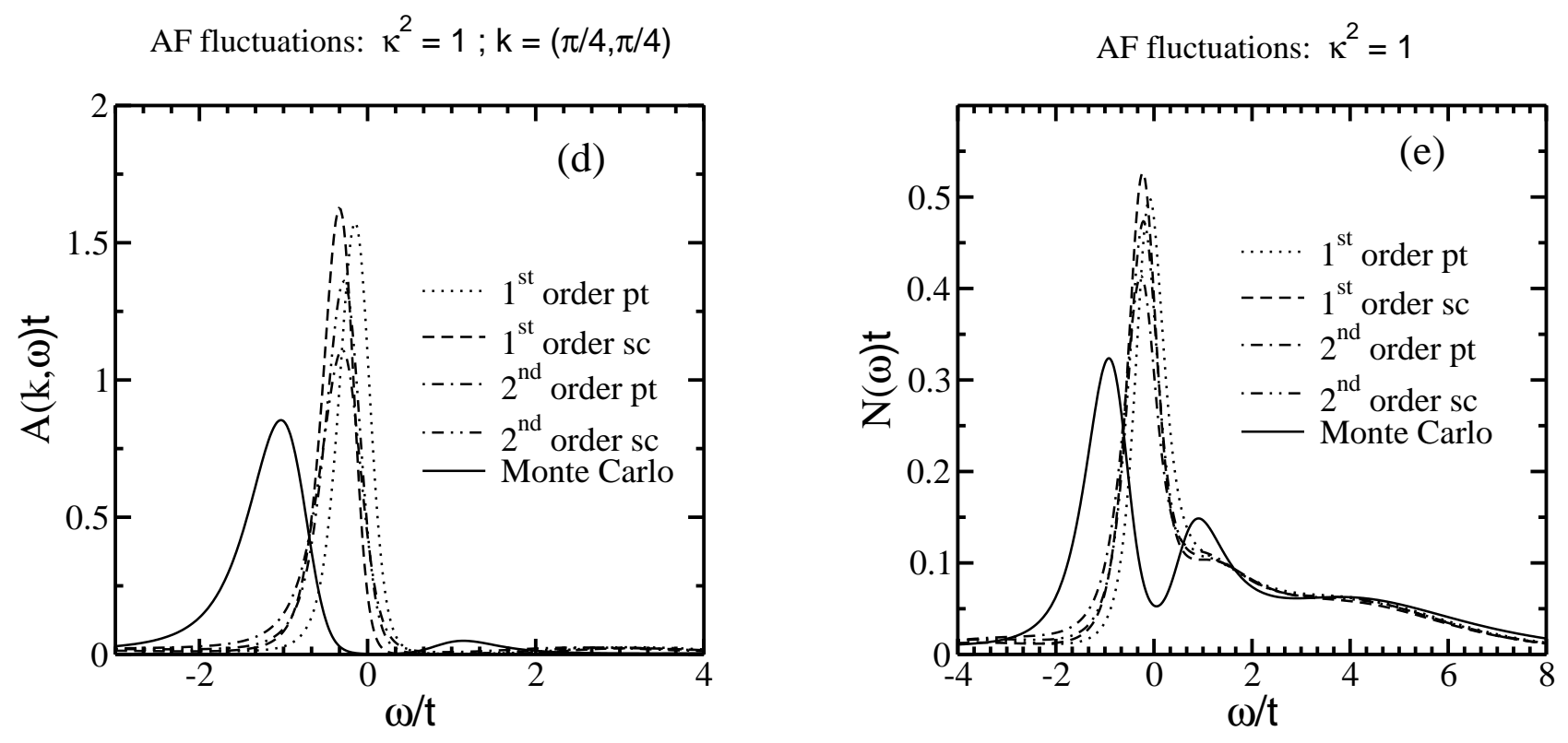

FIG. 5: The various perturbation-theoretic approximations to the quasiparticle imaginary time Green's function $\mathcal{G}(\mathbf{k}, \tau)$, spectral function $A(\mathbf{k}, \omega)$ and tunneling density of states $N(\omega)$ are compared to the results of the Monte Carlo simulations for $\kappa^{2}=1$ in the case of quasiparticles coupled to antiferromagnetic spin-fluctuations. $1^{\text {st }}$ order pt corresponds to the approximation to the self-energy shown in Fig. 2a and given by Eq. 25. $2^{\text {nd }}$ order pt corresponds to the approximation to the self-energy shown in Fig. 2c and given by Eq. (27). $1^{\text {st }}$ order sc corresponds to the approximation to the self-energy shown in Fig. 2b and given by Eq. (26). $2^{\text {nd }}$ order sc corresponds to the approximation to the self-energy shown in Fig. $2 \mathrm{~d}$ and given by Eq. (28). The error bars on the Monte Carlo imaginary time Green's function are not shown for clarity. They are of the order of $0.001 \mathrm{t}$. (a) $\mathcal{G}(\mathbf{k}, \omega)$ at $\mathbf{k}=(\pi, 0)$. (b) $A(\mathbf{k}, \omega)$ at $\mathbf{k}=(\pi, 0)$. (c) $\mathcal{G}(\mathbf{k}, \omega)$ at $\mathbf{k}=(\pi / 4, \pi / 4)$. (d) $A(\mathbf{k}, \omega)$ at $\mathbf{k}=(\pi / 4, \pi / 4)$. (e) $N(\omega)$. 
FE fluctuations: $\kappa^{2}=24 ; \mathrm{k}=(\pi, 0)$

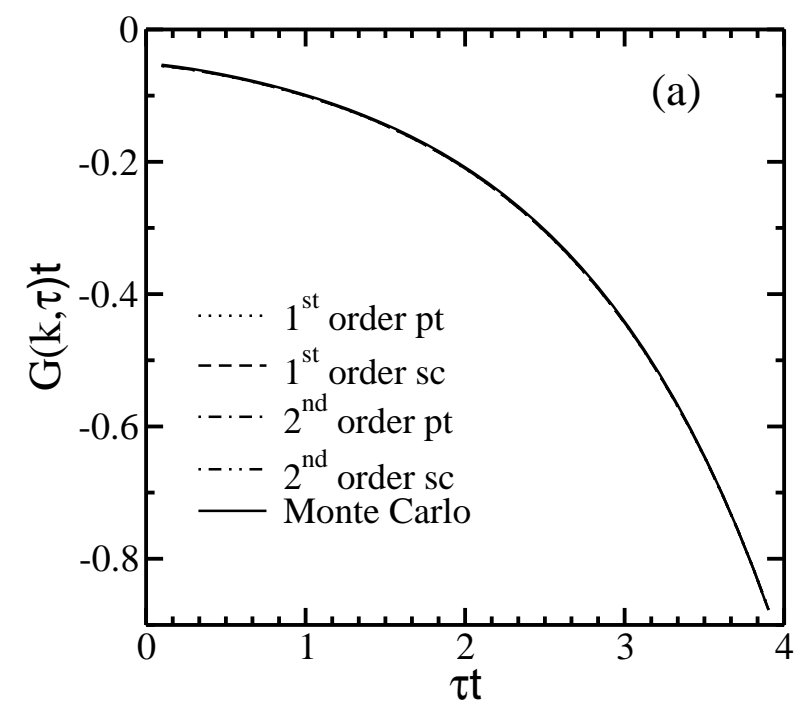

FE fluctuations: $\kappa^{2}=24 ; \mathrm{k}=(\pi, 0)$

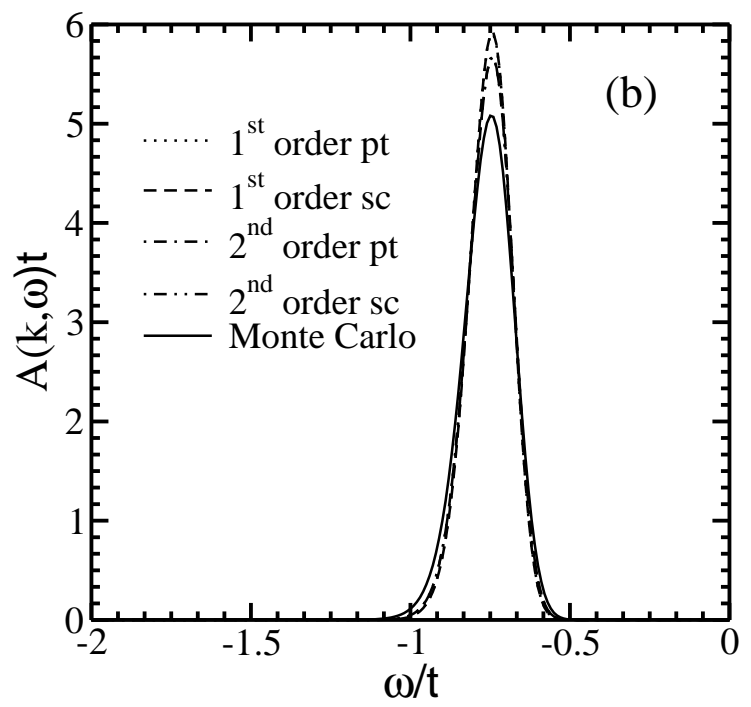




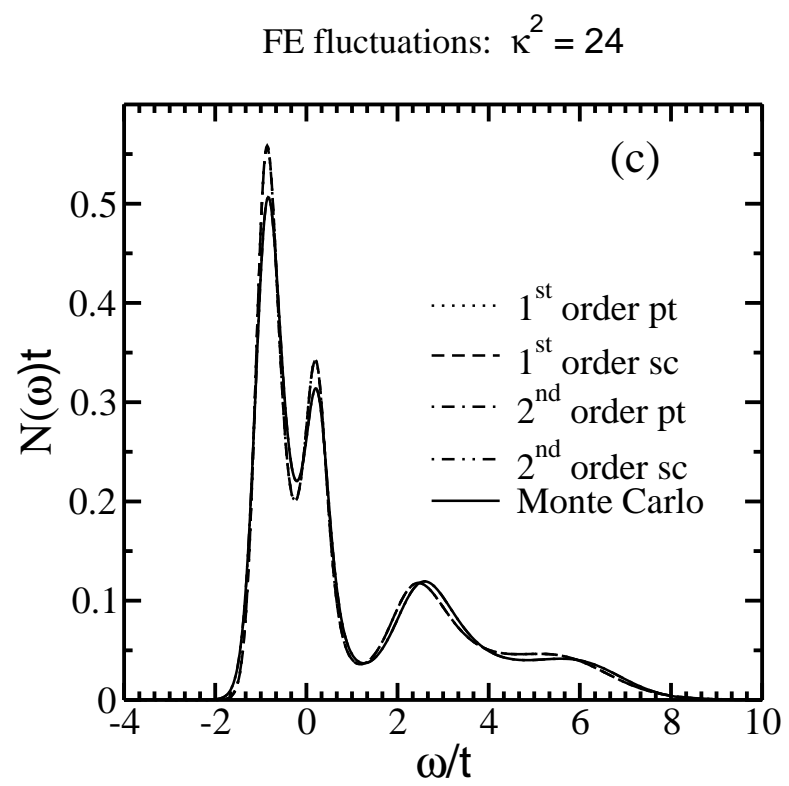

FIG. 6: The various perturbation-theoretic approximations to the quasiparticle imaginary time Green's function $\mathcal{G}(\mathbf{k}, \tau)$, spectral function $A(\mathbf{k}, \omega)$ and tunneling density of states $N(\omega)$ are compared to the results of the Monte Carlo simulations for $\kappa^{2}=24$ in the case of quasiparticles coupled to ferromagnetic spin-fluctuations. $1^{\text {st }}$ order pt corresponds to the approximation to the self-energy shown in Fig. 2a and given by Eq. (25). $2^{\text {nd }}$ order pt corresponds to the approximation to the selfenergy shown in Fig. 2c and given by Eq. (27). $1^{\text {st }}$ order sc corresponds to the approximation to the self-energy shown in Fig. $2 \mathrm{~b}$ and given by Eq. (26). $2^{\text {nd }}$ order sc corresponds to the approximation to the self-energy shown in Fig. 2d and given by Eq. (28). The error bars on the Monte Carlo imaginary time Green's function are not shown for clarity. They are of the order of 0.00002 t. (a) $\mathcal{G}(\mathbf{k}, \omega)$ at $\mathbf{k}=(\pi, 0)$. (b) $A(\mathbf{k}, \omega)$ at $\mathbf{k}=(\pi, 0)$. (c) $N(\omega)$. 
FE fluctuations: $\kappa^{2}=4 ; k=(\pi, 0)$

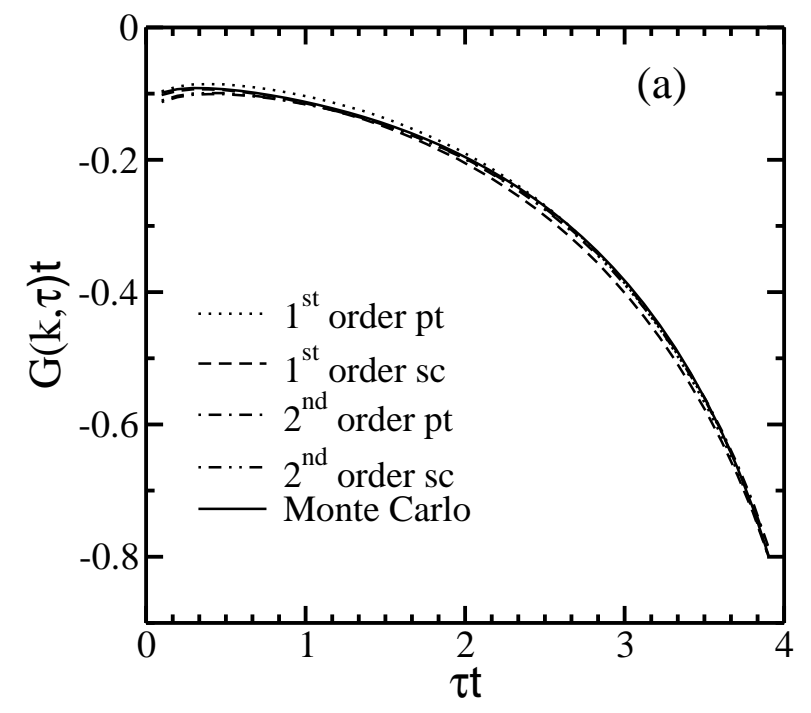

FE fluctuations: $\kappa^{2}=4 ; \mathrm{k}=(\pi, 0)$

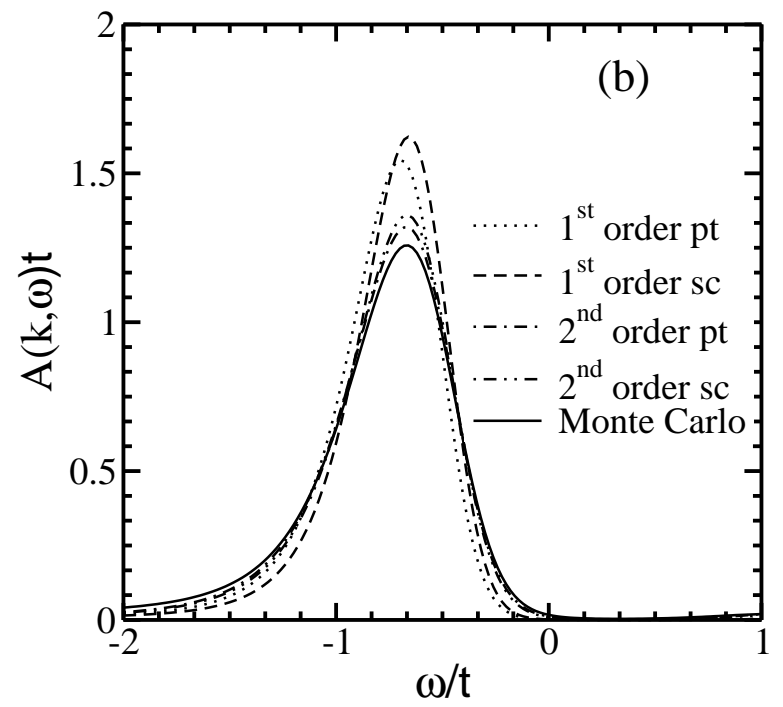




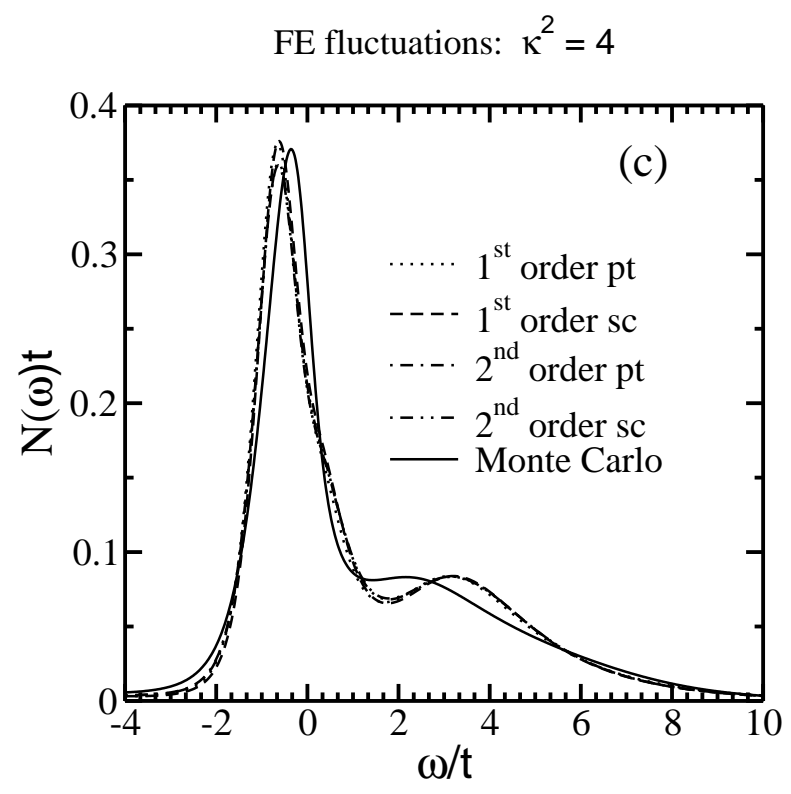

FIG. 7: The various perturbation-theoretic approximations to the quasiparticle imaginary time Green's function $\mathcal{G}(\mathbf{k}, \tau)$, spectral function $A(\mathbf{k}, \omega)$ and tunneling density of states $N(\omega)$ are compared to the results of the Monte Carlo simulations for $\kappa^{2}=4$ in the case of quasiparticles coupled to ferromagnetic spin-fluctuations. $1^{\text {st }}$ order pt corresponds to the approximation to the self-energy shown in Fig. 2a and given by Eq. (25). $2^{\text {nd }}$ order pt corresponds to the approximation to the selfenergy shown in Fig. 2c and given by Eq. 27. $1^{\text {st }}$ order sc corresponds to the approximation to the self-energy shown in Fig. $2 \mathrm{~b}$ and given by Eq. (26). $2^{\text {nd }}$ order sc corresponds to the approximation to the self-energy shown in Fig. 2d and given by Eq. (28). The error bars on the Monte Carlo imaginary time Green's function are not shown for clarity. They are of the order of $0.0002 \mathrm{t}$. (a) $\mathcal{G}(\mathbf{k}, \omega)$ at $\mathbf{k}=(\pi, 0)$. (b) $A(\mathbf{k}, \omega)$ at $\mathbf{k}=(\pi, 0)$. (c) $N(\omega)$. 
FE fluctuations: $\kappa^{2}=1 ; k=(\pi, 0)$

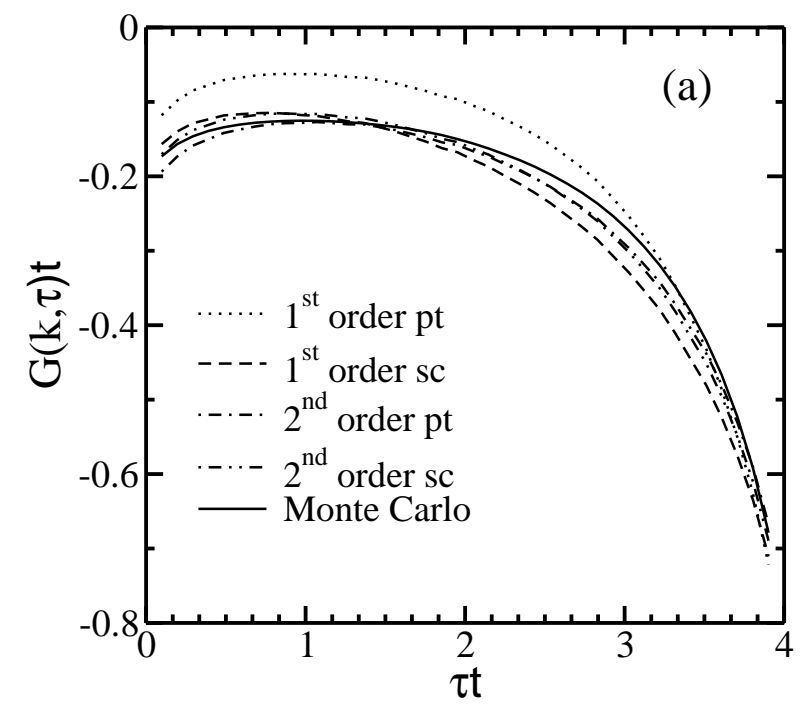

FE fluctuations: $\kappa^{2}=1 ; \mathrm{k}=(\pi, 0)$

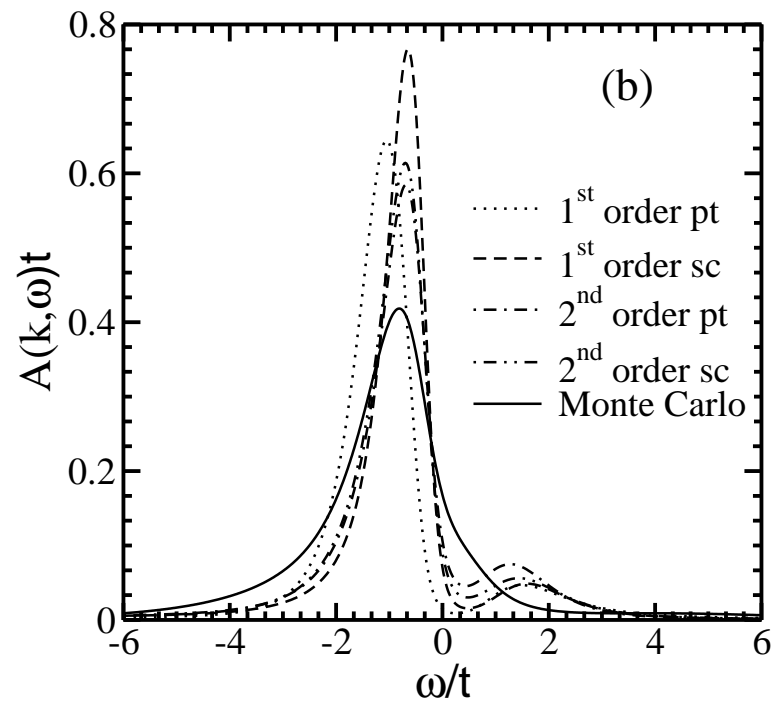




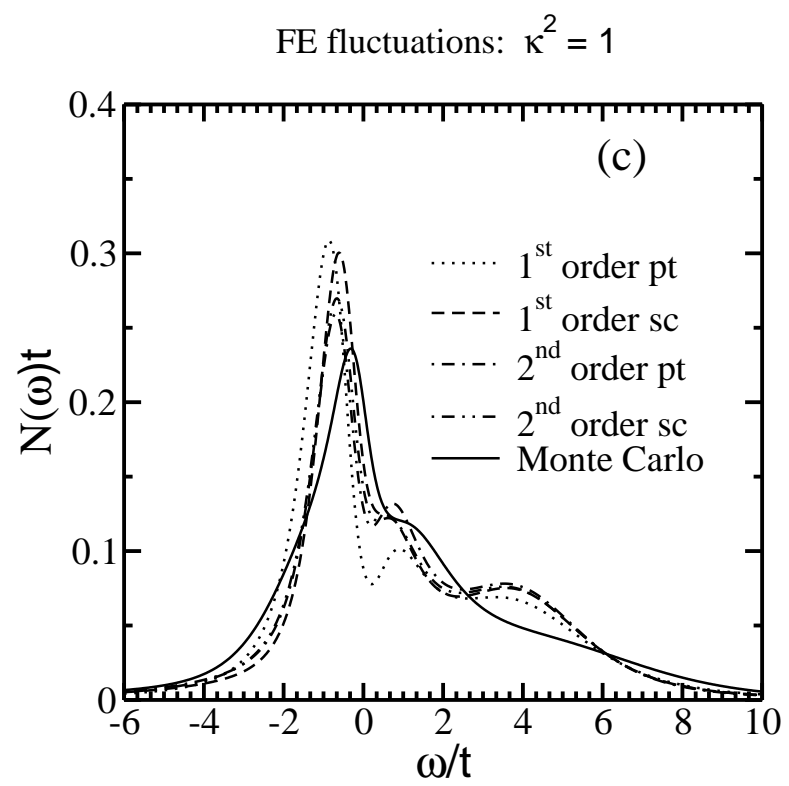

FIG. 8: The various perturbation-theoretic approximations to the quasiparticle imaginary time Green's function $\mathcal{G}(\mathbf{k}, \tau)$, spectral function $A(\mathbf{k}, \omega)$ and tunneling density of states $N(\omega)$ are compared to the results of the Monte Carlo simulations for $\kappa^{2}=1$ in the case of quasiparticles coupled to ferromagnetic spin-fluctuations. $1^{\text {st }}$ order pt corresponds to the approximation to the self-energy shown in Fig. 2a and given by Eq. (25). $2^{\text {nd }}$ order pt corresponds to the approximation to the selfenergy shown in Fig. 2c and given by Eq. 27. $1^{\text {st }}$ order sc corresponds to the approximation to the self-energy shown in Fig. 2b and given by Eq. (26). $2^{\text {nd }}$ order sc corresponds to the approximation to the self-energy shown in Fig. 2d and given by Eq. (28). The error bars on the Monte Carlo imaginary time Green's function are not shown for clarity. They are of the order of $0.0008 \mathrm{t}$. (a) $\mathcal{G}(\mathbf{k}, \omega)$ at $\mathbf{k}=(\pi, 0)$. (b) $A(\mathbf{k}, \omega)$ at $\mathbf{k}=(\pi, 0)$. (c) $N(\omega)$. 
FE fluctuations: $\kappa^{2}=0.25 ; \mathrm{k}=(\pi, 0)$

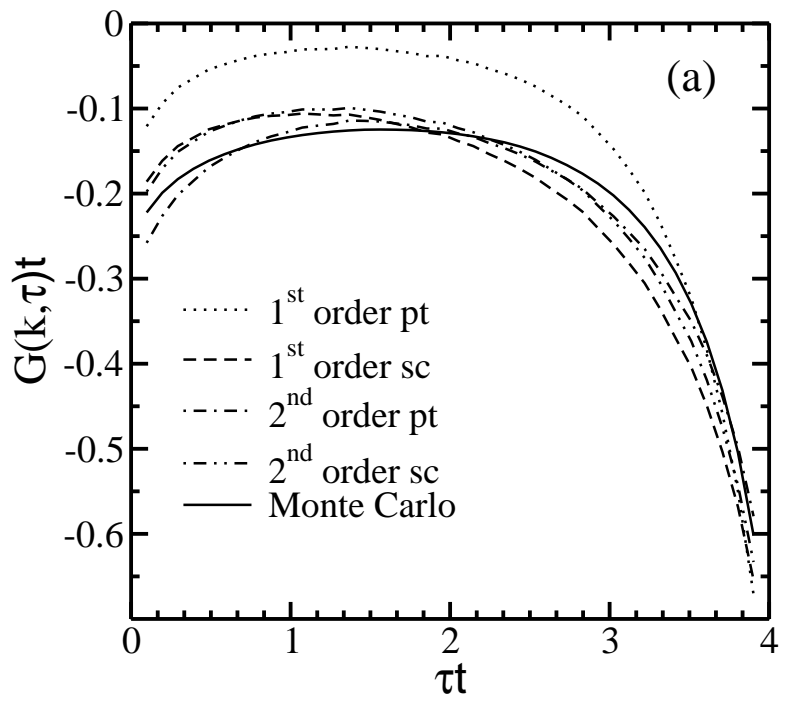

FE fluctuations: $\kappa^{2}=0.25 ; \mathrm{k}=(\pi, 0)$

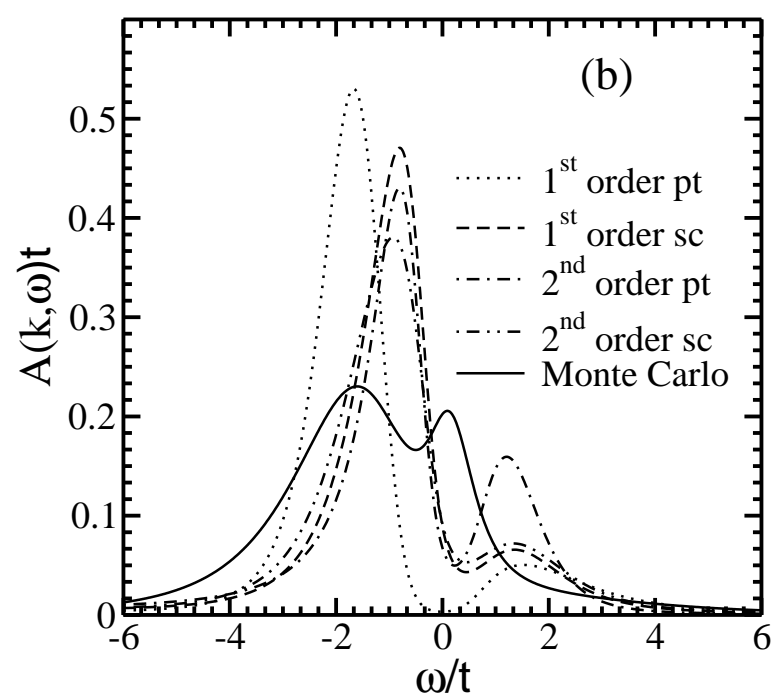

FE fluctuations: $\kappa^{2}=0.25 ; \mathrm{k}=(\pi / 4, \pi / 4)$

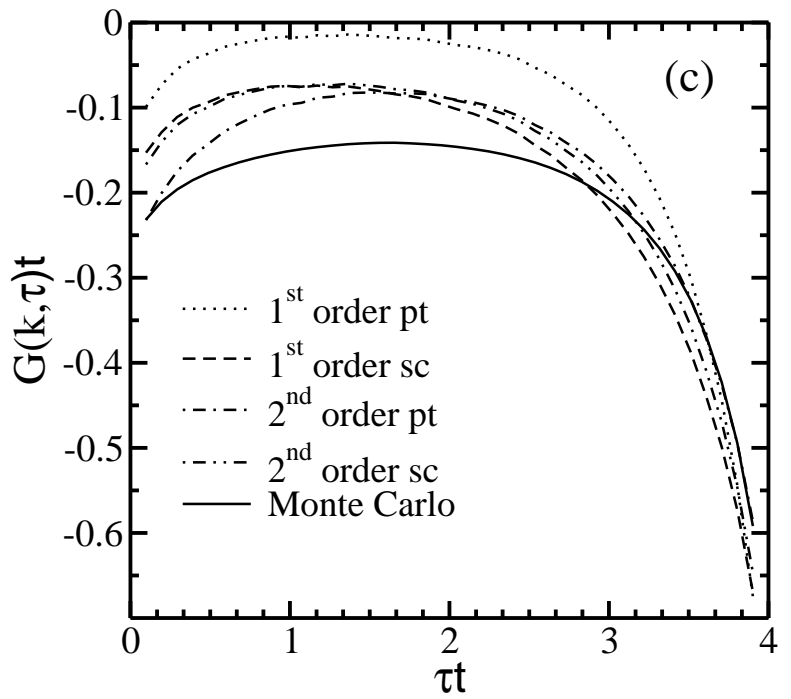


FE fluctuations: $\kappa^{2}=0.25 ; \mathrm{k}=(\pi / 4, \pi / 4)$

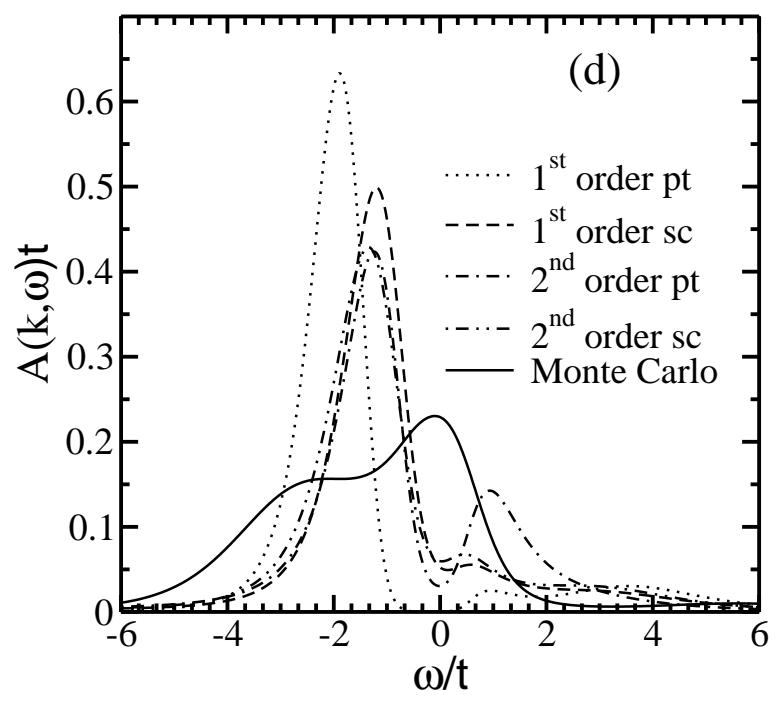

FE fluctuations: $\kappa^{2}=0.25$

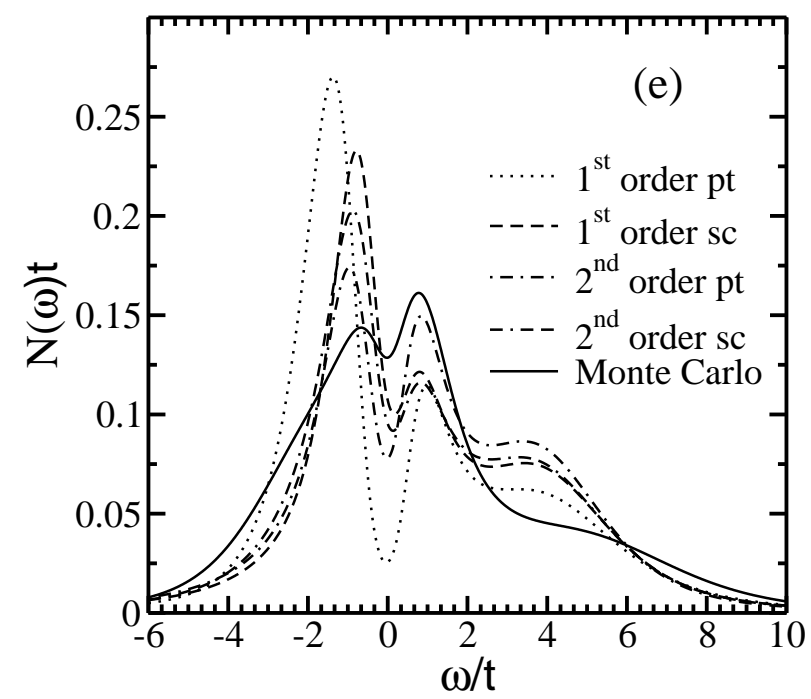

FIG. 9: The various perturbation-theoretic approximations to the quasiparticle imaginary time Green's function $\mathcal{G}(\mathbf{k}, \tau)$, spectral function $A(\mathbf{k}, \omega)$ and tunneling density of states $N(\omega)$ are compared to the results of the Monte Carlo simulations for $\kappa^{2}=0.25$ in the case of quasiparticles coupled to ferromagnetic spin-fluctuations. $1^{\text {st }}$ order pt corresponds to the approximation to the self-energy shown in Fig. 2a and given by Eq. 25. $2^{\text {nd }}$ order pt corresponds to the approximation to the self-energy shown in Fig. 2c and given by Eq. (27). $1^{\text {st }}$ order sc corresponds to the approximation to the self-energy shown in Fig. 2b and given by Eq.(26). $2^{\text {nd }}$ order sc corresponds to the approximation to the self-energy shown in Fig. $2 \mathrm{~d}$ and given by Eq. (28). The error bars on the Monte Carlo imaginary time Green's function are not shown for clarity. They are of the order of $0.001 \mathrm{t}$. (a) $\mathcal{G}(\mathbf{k}, \omega)$ at $\mathbf{k}=(\pi, 0)$. (b) $A(\mathbf{k}, \omega)$ at $\mathbf{k}=(\pi, 0)$. (c) $\mathcal{G}(\mathbf{k}, \omega)$ at $\mathbf{k}=(\pi / 4, \pi / 4)$. (d) $A(\mathbf{k}, \omega)$ at $\mathbf{k}=(\pi / 4, \pi / 4)$. (e) $N(\omega)$. 

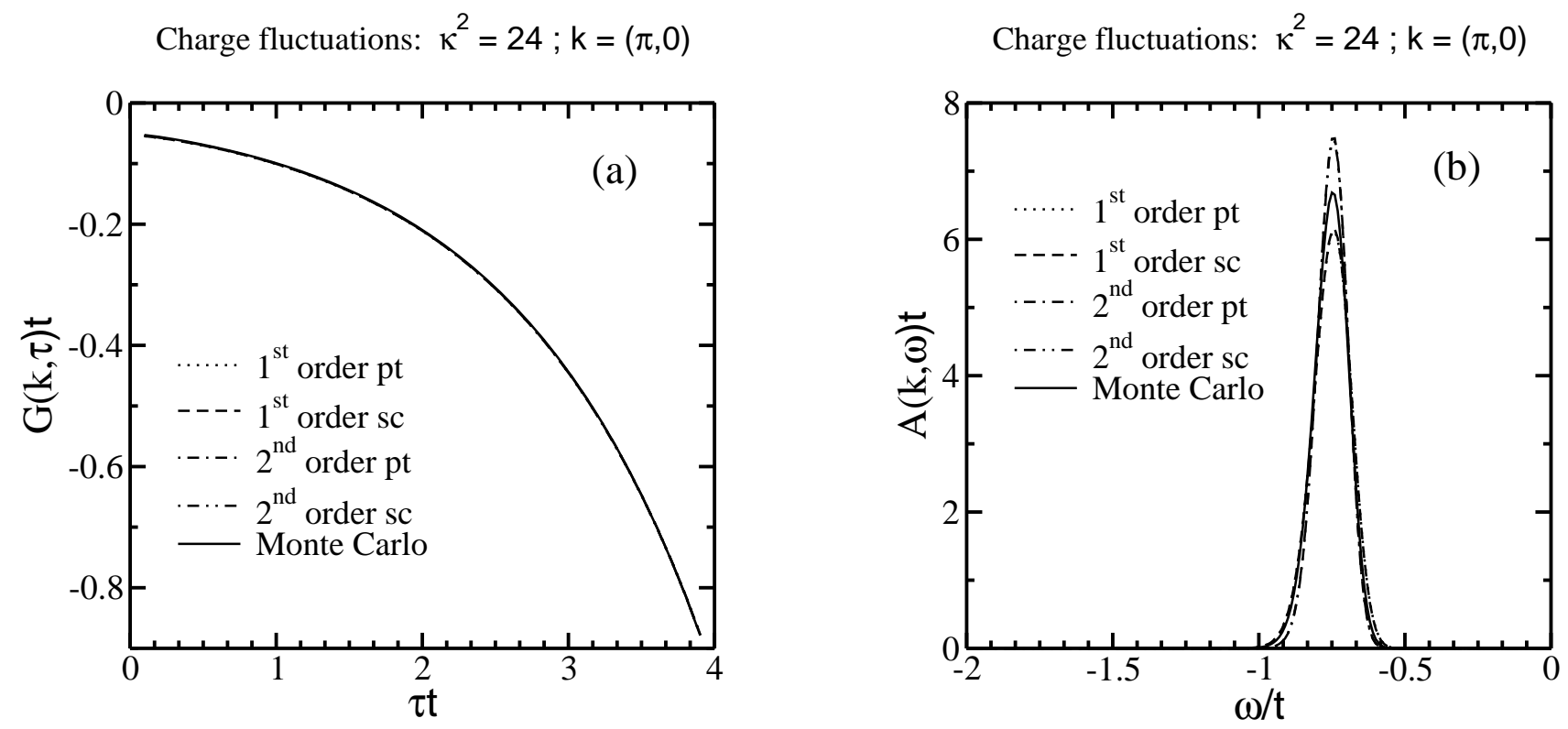


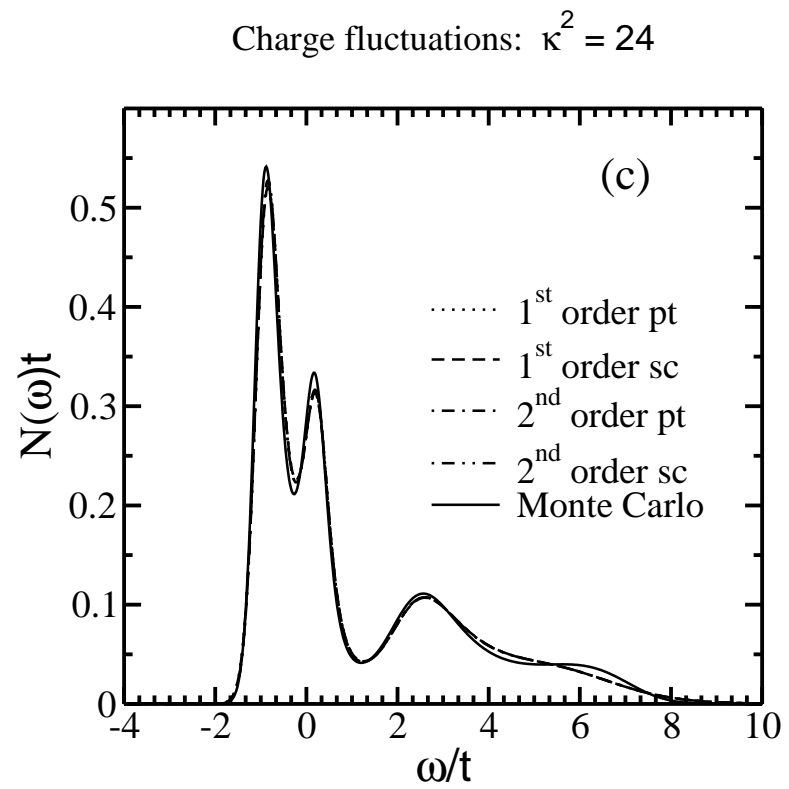

FIG. 10: The various perturbation-theoretic approximations to the quasiparticle imaginary time Green's function $\mathcal{G}(\mathbf{k}, \tau)$, the spectral function $A(\mathbf{k}, \omega)$ and tunneling density of states $N(\omega)$ are compared to the results of the Monte Carlo simulations for $\kappa^{2}=24$ in the case of quasiparticles coupled to charge fluctuations. $1^{\text {st }}$ order pt corresponds to the approximation to the self-energy shown in Fig. 2a and given by Eq. 25. $1^{\text {st }}$ order sc corresponds to the approximation to the self-energy shown in Fig. 2b and given by Eq. (26). The error bars on the Monte Carlo imaginary time Green's function are not shown for clarity. They are of the order of $0.00002 \mathrm{t}$. (a) $\mathcal{G}(\mathbf{k}, \omega)$ at $\mathbf{k}=(\pi, 0)$. (b) $A(\mathbf{k}, \omega)$ at $\mathbf{k}=(\pi, 0)$. (c) $N(\omega)$. 

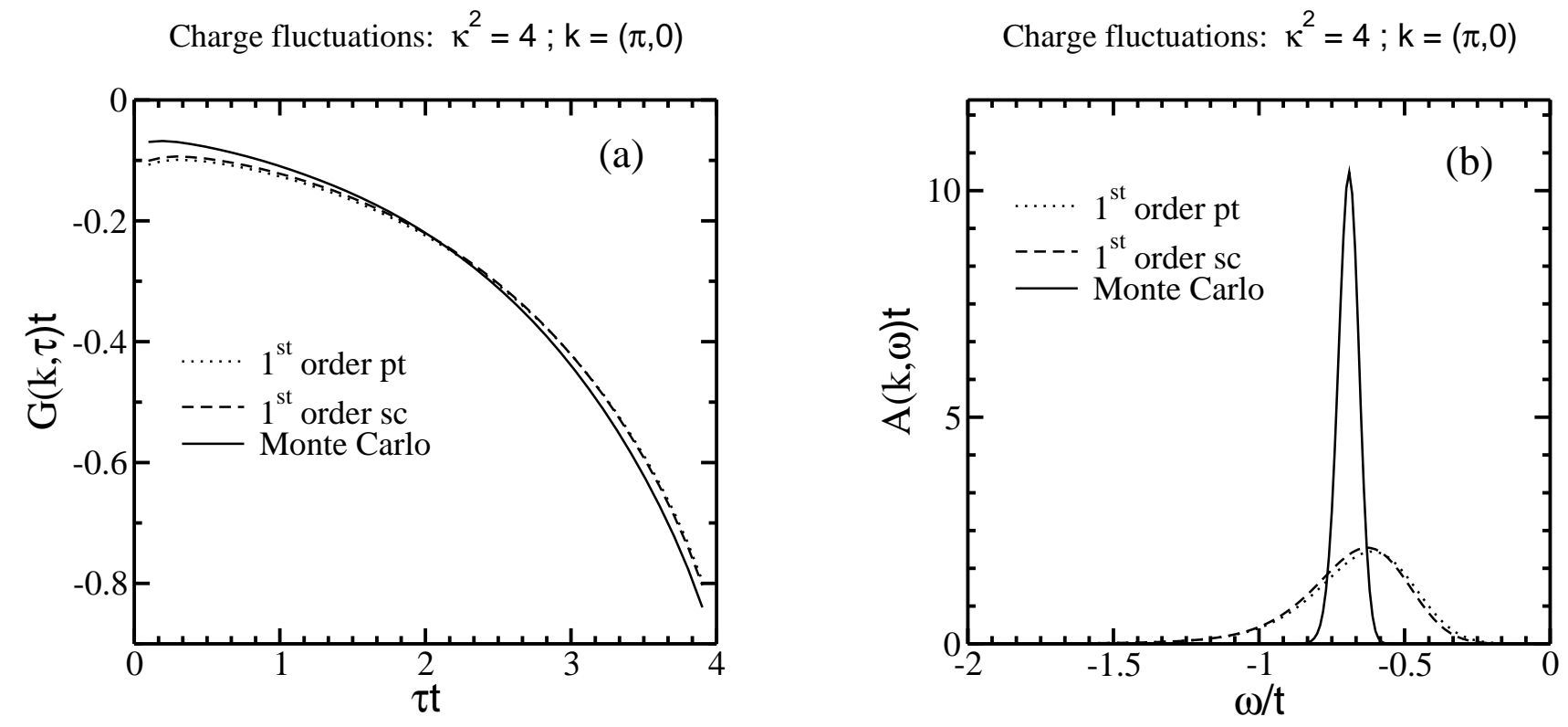


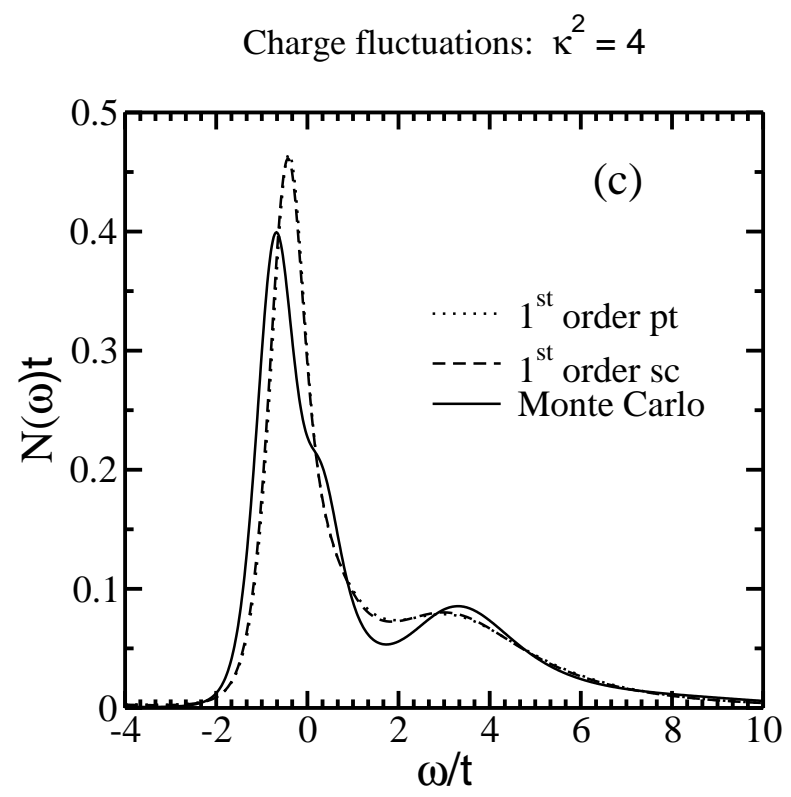

FIG. 11: The various perturbation-theoretic approximations to the quasiparticle imaginary time Green's function $\mathcal{G}(\mathbf{k}, \tau)$, the spectral function $A(\mathbf{k}, \omega)$ and tunneling density of states $N(\omega)$ are compared to the results of the Monte Carlo simulations for $\kappa^{2}=4$ in the case of quasiparticles coupled to charge fluctuations. $1^{\text {st }}$ order pt corresponds to the approximation to the self-energy shown in Fig. 2a and given by Eq. [25]. $1^{\text {st }}$ order sc corresponds to the approximation to the self-energy shown in Fig. 2b and given by Eq. (26). The error bars on the Monte Carlo imaginary time Green's function are not shown for clarity. They are of the order of $0.0002 \mathrm{t}$. (a) $\mathcal{G}(\mathbf{k}, \omega)$ at $\mathbf{k}=(\pi, 0)$. (b) $A(\mathbf{k}, \omega)$ at $\mathbf{k}=(\pi, 0)$. (c) $N(\omega)$. 
Charge fluctuations: $\kappa^{2}=0.25 ; \mathrm{k}=(\pi, 0)$

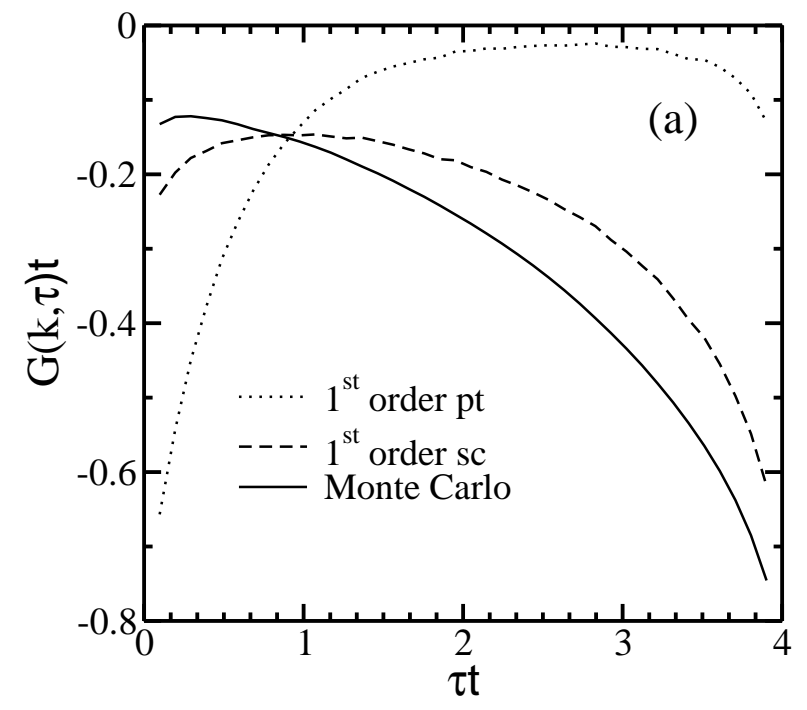

Charge fluctuations: $\kappa^{2}=0.25 ; \mathrm{k}=(\pi, 0)$

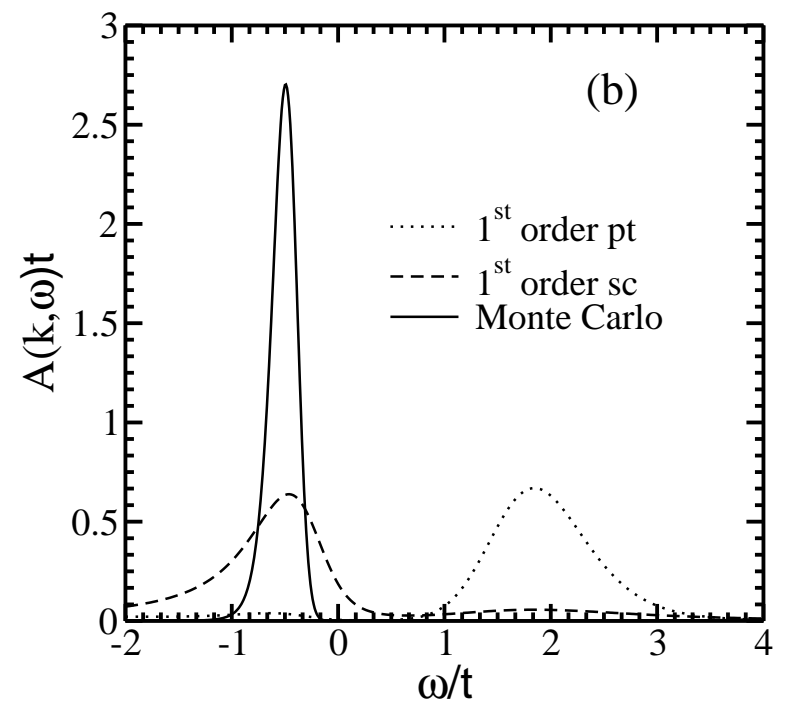

Charge fluctuations: $\kappa^{2}=0.25 ; \mathrm{k}=(\pi / 4, \pi / 4)$

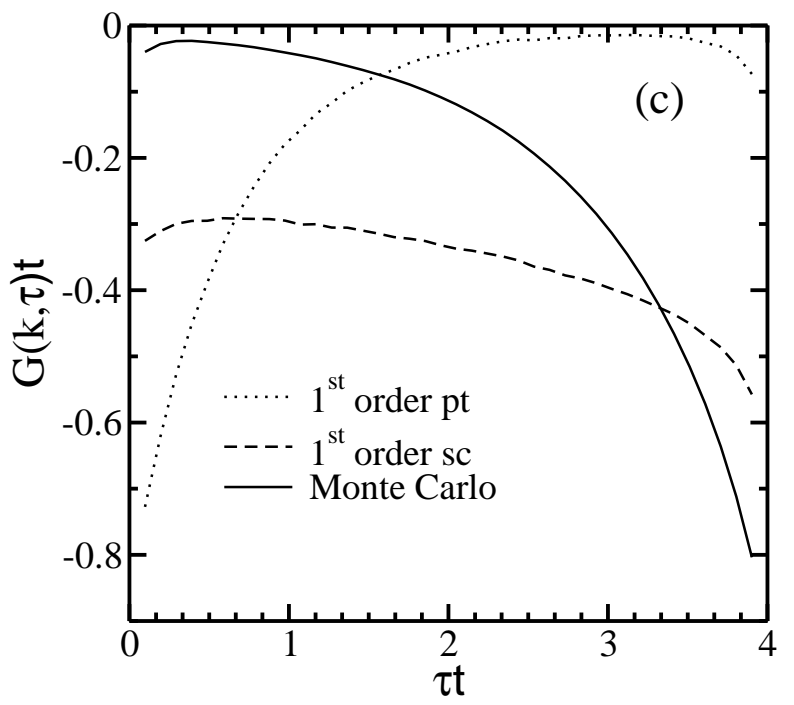


Charge fluctuations: $\kappa^{2}=0.25 ; \mathrm{k}=(\pi / 4, \pi / 4)$

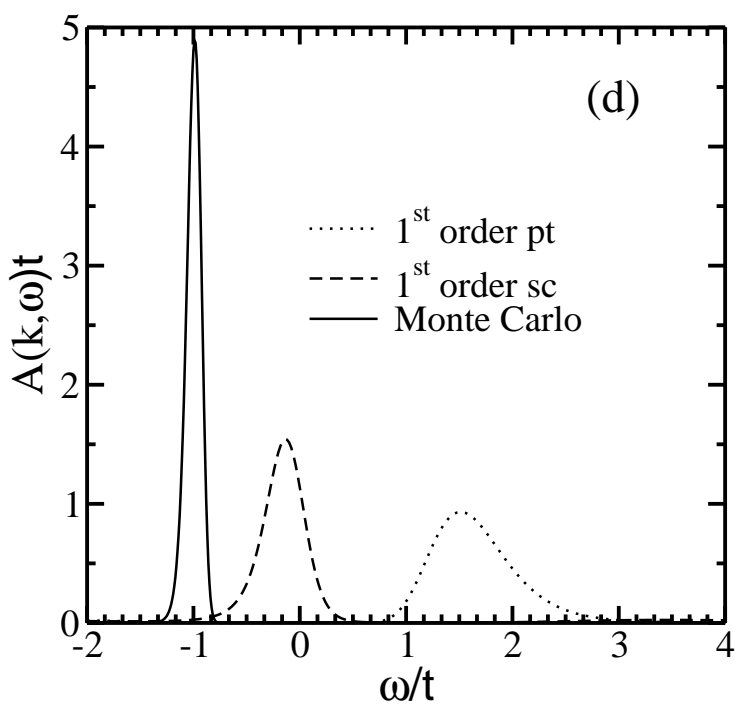

FIG. 12: The various perturbation-theoretic approximations to the quasiparticle imaginary time Green's function $\mathcal{G}(\mathbf{k}, \tau)$, the spectral function $A(\mathbf{k}, \omega)$ are compared to the results of the Monte Carlo simulations for $\kappa^{2}=0.25$ in the case of quasiparticles coupled to charge fluctuations. $1^{\text {st }}$ order pt corresponds to the approximation to the self-energy shown in Fig. 2a and given by Eq. (25). $1^{\text {st }}$ order sc corresponds to the approximation to the self-energy shown in Fig. $2 \mathrm{~b}$ and given by Eq.(26). The error bars on the Monte Carlo imaginary time Green's function are not shown for clarity. They are of the order of $0.002 \mathrm{t}$. (a) $\mathcal{G}(\mathbf{k}, \omega)$ at $\mathbf{k}=(\pi, 0)$. (b) $A(\mathbf{k}, \omega)$ at $\mathbf{k}=(\pi, 0)$. (c) $\mathcal{G}(\mathbf{k}, \omega)$ at $\mathbf{k}=(\pi / 4, \pi / 4)$. (d) $A(\mathbf{k}, \omega)$ at $\mathbf{k}=(\pi / 4, \pi / 4)$. 